\title{
Study of gauged lepton symmetry signatures at colliders
}

\author{
We-Fu Chang ${ }^{1,2, *}$ and John N. $\mathrm{Ng}^{2}$ \\ ${ }^{1}$ Department of Physics, National Tsing Hua University, Hsinchu 30013, Taiwan \\ ${ }^{2}$ Theory Group, TRIUMF, Vancouver, British Columbia V6T2A3, Canada
}

(Received 30 May 2018; published 9 August 2018)

\begin{abstract}
We construct a new gauged $U(1)_{\mathfrak{l}}$ lepton-number model that is anomaly-free for each standard model generation. The active neutrino masses are radiatively generated with a minimal scalar sector. The phenomenology and collider signals are studied. The interference effects among the new gauge boson, $Z_{\ell}$, photon, and $Z$ boson can be probed at the future $e^{+} e^{-}$colliders even if the center-of-mass energy is below the mass of $Z_{\ell}$. Moreover, the electroweak precision sets a stringent bound on the mass splitting of the new lepton doublets.
\end{abstract}

DOI: 10.1103/PhysRevD.98.035015

\section{INTRODUCTION}

It is well known that the standard model (SM) Lagrangian has an accidental global symmetry associated with the conservation of total lepton number. Equally well known is that the minimal SM cannot accommodate the evidence of active neutrino masses from the neutrino oscillations data. If one allows the dimension-5 Weinberg operator (Wo) [1] of the form $\frac{c}{\Lambda} L L H H$, where $L$ is the lefthanded lepton doublet, $H$ denotes the Higgs field, $\Lambda$ is an unknown high scale, and $c$ is a free parameter, then after spontaneous symmetry in which $H$ takes on a vacuum expectation value $v \simeq 247 \mathrm{GeV}$, we get a neutrino mass $m_{\nu} \sim \frac{c v^{2}}{\Lambda}$. Since data indicate that $m_{\nu} \lesssim 1 \mathrm{eV}$, the scale $\Lambda$ can range from 1 to $10^{11} \mathrm{TeV}$ depending on the value of $c$. If the neutrinos masses do indeed originate from the Weinberg operator, it fortifies the view that the SM is an effective field theory with a small violation of total lepton number in the form of the nonrenormalizable Wo.

The Wo gives an elegant explanation for neutrinos masses within the SM. However, its origin is not known and is the subject of the vast field of neutrino mass models. Furthermore, whether the lepton number is a global symmetry or a gauged symmetry and how the symmetry is broken are both open questions. The answers or even partial answers to these questions will add immensely to our understanding of fundamental physics. The simplest way to extend the SM and obtain the Wo is to have two or more SM singlet right-handed $(\mathrm{RH})$ neutrinos $N_{R}$. These

*wfchang@phys.nthu.edu.tw

Published by the American Physical Society under the terms of the Creative Commons Attribution 4.0 International license. Further distribution of this work must maintain attribution to the author(s) and the published article's title, journal citation, and DOI. Funded by SCOAP ${ }^{3}$. singlet neutrinos can be given heavy Majorana mass terms that change two units of lepton number explicitly by hand. Integrating these fields out will yield the Wo at low energies, which is the well-known type-I seesaw mechanism [2]. Instead of adding Majorana masses by hand, it is theoretically and phenomenologically more interesting to generate them by the spontaneous symmetry-breaking (SSB) mechanism. To this end, one adds a SM singlet scalar field $\Phi$ to construct the Yukawa term $\Phi \bar{N}_{R}^{c} N_{R}$. When $\Phi$ gets a vacuum expectation value (VEV) $\langle\Phi\rangle \gg v$, one again gets the type-I seesaw. If the lepton symmetry that is broken is a $U(1)$ global symmetry, then a singlet scalar Majoron will exist in the physical spectrum and can act as extra dark radiation [3]. An extended model with a dark matter candidate has also been constructed in Refs. [4,5]. Moreover, this symmetry can also be a local gauge symmetry.

The study of the lepton number being a local gauge symmetry has long history. If the symmetry were unbroken, one would have a leptonic photon [6]. The corresponding long-range force can be searched for in equivalence principle tests [7], and the limit on the leptonic fine structure constant is $\alpha_{l}<10^{-49}$. However, a complete and consistent model was not studied until recently in Ref. [8] in conjunction with the gauged baryon number. Gauging the lepton number only is given in Ref. [9], in which active neutrino masses are given by the usual type-I seesaw model. A different implementation with the type-II seesaw mechanism [10] is given in Ref. [11]. Also, there, the emphasis is on constructing a consistent dark matter model with a gauged lepton number. More recently, a gauged $S U(2)_{\ell}$ model was considered by Ref. [12] with an emphasis on producing a dark matter candidate and baryogenesis.

In this work, we study a model of gauged lepton number $U(1)_{\mathfrak{r}}$ without employing the type-I seesaw mechanism for active neutrino masses. Specifically, the model does not 
have SM singlet neutrinos. The SM leptons are assigned lepton number $\ell=1$, and $U(1)_{\mathfrak{l}}$ is spontaneously broken. The existence of lepton specific gauge boson $Z_{\mathfrak{l}}$ is a robust prediction of this class of models. The SM is anomalous under $U(1)_{\mathfrak{r}}$, and hence new chiral fermions will have to be added. Our solution differs from that of Ref. [9], in which the authors use a set a fermions to solve the anomalies from all three SM lepton families together. We choose to solve the anomaly of the SM leptons within each family, and we do not have SM singlet neutrinos as mentioned before.

It is well known that, given two $U(1)$ gauge symmetries, their corresponding gauge bosons can have kinetic mixing [13] as well as mass mixing. Both are expected to be small. The phenomenology of a kinetically mixed $Z^{\prime}$ with a $U(1)_{Y}$ gauge boson was given in Ref. [14]. In this paper, we shall neglect these mixings.

This paper is organized as follows. In Sec. II, we discuss the anomalies cancelation solution and the new chiral fermions. Section III constructs the Yukawa interactions and the minimal set of scalars required. The scalar potential that leads to symmetry breaking and the charged lepton masses of the model are also constructed and studied. The extended gauge interactions are described in Sec. IV. Particular attention is given to $Z_{\mathfrak{l}}$ which must exist in these models, independent of which solution to the anomalies one adopts. It is natural to assume that all the SM charged leptons carry one unit of lepton number. Moreover, the rich phenomenology of $Z_{l}$ at the past and future lepton colliders is guaranteed. Even if its mass denoted by $M_{X}$ is too heavy to be produced at these colliders its interference with the SM $\gamma$ and $Z$ can be detected in precision measurements. Such effects are proportional to $\frac{1}{M_{X}^{2}}$ and thus sensitive to low mass $Z_{\mathfrak{r}}$. These are discussed in Sec. V. The production at the LHC is also given there. Since $Z_{\mathfrak{l}}$ couples only to leptons and not quarks, the search strategy will have to be different from the usual extra $Z$ boson searches. Active neutrino masses are generated by 1-loop effect and is given in Sec. VI. Since it is not the purpose of this paper to do detail neutrino oscillation study we will only present orders of magnitude estimates. This is followed by a discussion of the phenomenology of the new fermions in Sec. VII. Our conclusions are given in Sec. VIII.

\section{ANOMALIES CANCELATIONS}

We extend the SM gauge group by $U(1)_{\mathfrak{r}}$; explicitly, it is $G=G_{\mathrm{SM}} \times U(1)_{\mathfrak{l}}=S U(2) \times U(1)_{Y} \times U(1)_{\mathfrak{l}}$. The color $S U(3)$ group plays no role here and can be neglected. We define the SM leptons to have number $\ell=1$ under $U(1)_{\mathfrak{r}}$. The new anomaly coefficients for a single SM lepton family are

$$
\begin{gathered}
\mathcal{A}_{1}\left([S U(2)]^{2} U(1)_{\mathfrak{l}}\right)=-1 / 2, \\
\mathcal{A}_{2}\left(\left[U(1)_{Y}\right]^{2} U(1)_{\mathfrak{l}}\right)=1 / 2, \\
\mathcal{A}_{3}\left(\left[U(1)_{Y}\left[U(1)_{\mathfrak{r}}\right]^{2}\right)=0,\right.
\end{gathered}
$$

$$
\begin{gathered}
\mathcal{A}_{4}\left(\left[U(1)_{\mathfrak{r}}\right]^{3}\right)=-1, \\
\mathcal{A}_{5}\left(U(1)_{\mathfrak{l}}\right)=-1,
\end{gathered}
$$

where $\mathcal{A}_{5}$ is for the lepton-graviton anomaly. We also need to check if the SM anomalies of $\mathcal{A}_{6}\left([S U(2)]^{2} U(1)_{Y}\right)$, $\mathcal{A}_{7}\left(\left[U(1)_{Y}\right]^{3}\right)$ and $\mathcal{A}_{8}\left(U(1)_{Y}\right)$ are canceled when new chiral leptons are introduced to cancel Eq. (1).

We introduce two sets of chiral leptons very similar to the SM leptons. The first set consists of an $S U(2)$ doublet and a singlet and has the eigenvalue $\ell_{1}$. Explicitly, we write

$$
L_{1 L}=\left(N_{1 L}, E_{1 L}\right) ; \quad\left[\mathbf{2},-\frac{1}{2}, \ell_{1}\right], \quad E_{1 R} ; \quad\left[\mathbf{1},-1, \ell_{1}\right],
$$

where the subscript $L(R)$ stands for left- (right-)handed projections. The square brackets [...] denote $S U(2), U(1)_{Y}$, $U_{\mathfrak{l}}$ assignments. A second set with right-handed projections but a lepton number of $\ell_{2}$ is given by

$L_{2 R}=\left(N_{2 R}, E_{2 R}\right) ; \quad\left[\mathbf{2},-\frac{1}{2}, \ell_{2}\right], \quad E_{2 L} ; \quad\left[\mathbf{1},-1, \ell_{2}\right]$.

It is easy to see that Eqs. (1) become

$$
\begin{aligned}
& \mathcal{A}_{1}=-\frac{1}{2}\left(\ell_{1}-\ell_{2}+1\right), \\
& \mathcal{A}_{2}=\frac{1}{2}\left(\ell_{1}-\ell_{2}+1\right), \\
& \mathcal{A}_{3}=0 \\
& \mathcal{A}_{4}=-\ell_{1}^{3}+\ell_{2}^{3}-1, \\
& \mathcal{A}_{5}=-\left(\ell_{1}-\ell_{2}+1\right) .
\end{aligned}
$$

$\mathcal{A}_{1,2,5}=0$ for $\ell_{2}=\ell_{1}+1$. Substituting into $\mathcal{A}_{4}=0$ gives

$$
\ell_{1}\left(\ell_{1}+1\right)=0
$$

The two solutions are:

(i) Solution I:

$$
\ell_{1}=-1 \text { and } \ell_{2}=0 .
$$

(ii) Solution II:

$$
\ell_{1}=0 \text { and } \ell_{2}=1 .
$$

It is easy to check that the solutions do not contribute to $\mathcal{A}_{6,7,8}$. This is not surprising since both Eqs. (2) and (3) form 
TABLE I. Lepton fields for anomaly-free solution I.

\begin{tabular}{lccr}
\hline \hline Field & $S U(2)$ & $Y$ & $\ell$ \\
\hline$\ell_{L}=\left(\begin{array}{l}\nu_{L} \\
e_{L}\end{array}\right)$ & $\mathbf{2}$ & $-\frac{1}{2}$ & 1 \\
$e_{R}$ & $\mathbf{1}$ & -1 & 1 \\
$L_{1 L}=\left(\begin{array}{c}N_{1 L} \\
E_{1 L}\end{array}\right)$ & $\mathbf{2}$ & $-\frac{1}{2}$ & -1 \\
$E_{1 R}$ & $\mathbf{1}$ & -1 & -1 \\
$L_{2 R}=\left(\begin{array}{l}N_{2 R} \\
E_{2 R}\end{array}\right)$ & $\mathbf{2}$ & $-\frac{1}{2}$ & 0 \\
$E_{2 L}$ & $\mathbf{1}$ & -1 & 0 \\
\hline \hline
\end{tabular}

vectorlike pairs under $G_{\mathrm{SM}}$. Thus, Eqs. (6) and (7) are anomaly-free without the use of singlet RH neutrinos.

\section{YUKAWA INTERACTIONS}

After determining the anomaly-free lepton representations, we can proceed to construct $G$-invariant Yukawa interactions. This will produce the minimal scalar fields required for viable charged and neutral lepton mass matrices at the tree level.

We will give a detailed discussion of the physics associated with solution I. ${ }^{1}$ The complete set of leptons for this solution and their gauge quantum numbers are given in Table I. With this, one can form all possible Lorentz-invariant bilepton combinations that are invariant under $G_{\mathrm{SM}}$. The next step is to identify scalar fields that will make Yukawa interactions that are invariant under the full gauge group $G$. Besides the SM Higgs field $H_{0}$, the minimum set of new scalars we require is $\Phi, S$, and $H_{1}$, and their quantum numbers are also given in Table II.
TABLE II. Minimal scalar fields for leptons of solution I.

\begin{tabular}{lccc}
\hline \hline Field & $S U(2)$ & $Y$ & $\ell$ \\
\hline$H_{0}=\left(\begin{array}{c}0 \\
\frac{v+h}{\sqrt{2}}\end{array}\right)$ & $\mathbf{2}$ & $\frac{1}{2}$ & 0 \\
$H_{1}=\left(\begin{array}{c}H_{1}^{+} \\
H_{1}^{+}\end{array}\right)$ & $\mathbf{2}$ & $\frac{1}{2}$ & 2 \\
$S$ & $\mathbf{1}$ & 1 & 0 \\
$\Phi_{1}$ & $\mathbf{1}$ & 0 & 1 \\
$\Phi_{2}$ & $\mathbf{1}$ & 0 & 2 \\
\hline \hline
\end{tabular}

With this, the Yukawa interactions are given $b y^{2}$

$$
\begin{aligned}
\mathcal{L}_{y}= & y_{e} \overline{\bar{\ell}_{L}} e_{R} H_{0}+Y_{2} \overline{L_{1 L}} E_{1 R} H_{0}+Y_{3} \overline{L_{2 R}} E_{2 L} H_{0} \\
& +\lambda_{1} \overline{\ell_{L}} L_{2 R} \Phi_{1}+\lambda_{2} \overline{E_{2 L}} e_{R} \Phi_{1}^{\dagger}+\lambda_{3} \overline{L_{1 L}} L_{2 R} \Phi_{1}^{*} \\
& +\lambda_{4} \overline{E_{2 L}} E_{1 R} \Phi_{1}+Y_{1} \overline{\ell_{L}} H_{1} E_{1 R}+f \overline{\ell_{L}^{c}} \epsilon L_{1 L} S+\text { H.c. }
\end{aligned}
$$

It can be seen that $S$ is charged and cannot develop a VEV. $H_{1}$ is a Higgs-like field and may or may not pick up a VEV depending on the parameters in the scalar potential. Here, we make the reasonable assumption that the lepton number-breaking scale is much higher than $v$. To not have a weak-scale lepton-number violation, we will work in the parameter space where $H_{1}$ is not Higgssed since it has $\ell=2$. Moreover, $\Phi_{1}$ is a neutral scalar, and it can pick up a VEV $w$ and thus can bestow masses to the new charged leptons $E_{1,2}$. They will be much heavier than SM charged leptons if $w \gg v$. Another electroweak singlet scalar $\Phi_{2}$ with 2 units of lepton number is required for neutrino mass generation, as we shall see later. But it does not enter in Eq. (8). We will not include scalar fields with $|Y|>1$ as they play no role in our study.

Having specified all the necessary scalars, the minimal $G$-invariant scalar potential is given by

$$
\begin{aligned}
V\left(H_{0}, H_{1}, \Phi_{1}, \Phi_{2}, S\right)= & -\mu^{2} H_{0}^{\dagger} H_{0}+m_{1}^{2} H_{1}^{\dagger} H_{1}+\kappa_{0}\left(H_{0}^{\dagger} H_{0}\right)^{2}+\kappa_{1}\left(H_{1}^{\dagger} H_{1}\right)^{2}+\kappa_{2}\left(H_{0}^{\dagger} H_{0}\right)\left(H_{1}^{\dagger} H_{1}\right)+\kappa_{3}\left(H_{0}^{\dagger} H_{1}\right)\left(H_{1}^{\dagger} H_{0}\right) \\
& +m_{S}^{2} S^{\dagger} S+\kappa_{S}\left(S^{\dagger} S\right)^{2}-\sum_{i=1,2} \mu_{i}^{2} \Phi_{i}^{\dagger} \Phi_{i}+\kappa_{11}\left(\Phi_{1}^{\dagger} \Phi_{1}\right)\left(\Phi_{1}^{\dagger} \Phi_{1}\right)+\kappa_{12}\left(\Phi_{1}^{\dagger} \Phi_{1}\right)\left(\Phi_{2}^{\dagger} \Phi_{2}\right)+\kappa_{22}\left(\Phi_{2}^{\dagger} \Phi_{2}\right)\left(\Phi_{2}^{\dagger} \Phi_{2}\right) \\
& +\sum_{i=1,2} \sum_{j=0,1} \kappa_{\Phi_{i} H_{j}}\left(\Phi_{i}^{\dagger} \Phi_{i}\right)\left(H_{j}^{\dagger} H_{j}\right)+\sum_{i=1,2} \kappa_{\Phi_{i} S}\left(\Phi_{i}^{\dagger} \Phi_{i}\right)\left(S^{\dagger} S\right) \\
& +\sum_{i=0,1,2} \kappa_{H_{i} S}\left(H_{i}^{\dagger} H_{i}\right)\left(S^{\dagger} S\right)+\lambda_{1 \ell} H_{1} \epsilon H_{0} S^{\dagger} \Phi_{2}^{\dagger}+\lambda_{2 \ell} H_{0}^{\dagger} H_{1}\left(\Phi_{1}^{*}\right)^{2}+\mu_{3} H_{0}^{\dagger} H_{1} \Phi_{2}^{*}+\mu_{4}\left(\Phi_{1}^{*}\right)^{2} \Phi_{2}+\text { H.c. }
\end{aligned}
$$

\footnotetext{
${ }^{1}$ Solution II gives qualitatively the same physics. It is easy to extend our discussions to this case.

${ }^{2} \mathrm{We}$ are interested in the minimal setup. In general there could also be a Yukawa term $\bar{L}_{1 L} e_{R} H_{2}$ with a second doublet $H_{2}:(2,1 / 2,-2)$.
}

Lepton-number violation occurs spontaneously for $\left\langle\left|\Phi_{1,2}\right|\right\rangle=w / \sqrt{2} \neq 0$ and is the only such scale in the model. ${ }^{3}$ Thus, we write $\Phi_{1(2)}=\frac{w+\varphi_{1,(2)}}{\sqrt{2}}$.

\footnotetext{
${ }^{3}$ In general, $\Phi_{1}$ and $\Phi_{2}$ need not have the same VEV. This only adds more parameters to the model without adding more physics. We shall assume they are equal.
} 
After SSB, the lepton mass matrices arise from Eq. (8). The charged lepton matrix in the basis $\mathcal{E}=\left(e_{w}, E_{1}, E_{2}\right)^{4}$ is

$$
M_{E}=\frac{w}{\sqrt{2}}\left(\begin{array}{ccc}
y_{e} r & 0 & \lambda_{2} \\
0 & Y_{2} r & \lambda_{4} \\
\lambda_{1} & \lambda_{3} & Y_{3} r
\end{array}\right),
$$

where $r \equiv \frac{v}{w} \ll 1$. And the neutral lepton mass matrix in terms of the chiral states $\left(\nu_{L}^{w}, N_{1 L}, N_{2 R}^{c}\right)^{5}$ is

$$
M_{N}=\frac{w}{\sqrt{2}}\left(\begin{array}{ccc}
0 & 0 & \lambda_{1} \\
0 & 0 & \lambda_{3} \\
\lambda_{1} & \lambda_{3} & 0
\end{array}\right) .
$$

The identity $\bar{\psi}_{1}^{c}(\hat{L} / \hat{R}) \psi_{2}^{c}=\bar{\psi}_{2}(\hat{L} / \hat{R}) \psi_{1}$ has been used to give the symmetric $M_{N}$, which is a tree-level result. At this level, the active neutrino is massless.

However, what is interesting is that Eq. (9) has sufficient structure to give a one-loop radiative Majorana mass to $\nu_{L}^{w}$; i.e., the upper leftmost entry of Eq. (11) will have a quantum contribution. The source comes from the term involving $\lambda_{2 \ell}$, which spontaneously breaks lepton symmetry when $\Phi_{2}$ gets a VEV. It also induces a mixing between the charged scalars $H_{1}^{+}$and $S^{+}$. The details of radiatively generated active neutrino masses will be discussed in a later section.

Equation (8) holds for a single lepton family. It can be generalized to the three-families case by promoting the couplings $y_{e}, Y_{1,2,3}, \lambda_{1, \ldots, 4}, f$ to $3 \times 3$ matrices. There are also similar terms connecting different families, which we will neglect since we are not interested in charged leptonflavor violation or flavor-changing neutral current processes in this paper. Henceforth, our discussions will mostly involve only a single lepton family, which is designated as the electron family.

\section{A. Quartet of scalar fields}

It is easy to see from Eq. (9) that the SM Higgs field in the gauge basis can be identified with $H_{0}$. The SM Higgs field will mix with the real parts of the SM singlets $\Re \Phi_{1,2}$ and SM doublet charge neutral part $\Re H_{1}^{0}$ through the quartic couplings $\lambda_{2 \ell}, \lambda_{\Phi_{1} H_{0}}, \lambda_{\Phi_{2} H_{0}}$ and the cubic term $\mu_{3}$ after SSB. The scalar mass matrix is in general $4 \times 4$. We denote this quartet of gauge states by $\mathcal{H}_{\alpha}=\frac{1}{\sqrt{2}}\left(\Re H_{0}^{0}, \Re H_{1}^{0}, \Re \Phi_{1}, \Re \Phi_{2}\right)$. As usual, the mass eigenstates $\mathfrak{h}_{i}=\left(h_{0}, h_{1}, h_{2}, h_{3}\right)$ are related to $\mathcal{H}$ via $\mathfrak{h}=U \mathcal{H}$, where $U$ is a $4 \times 4$ unitary mixing matrix. The strength of the mixings given by the elements $U_{i \alpha}$ will depend on the physical masses of the new scalars and the

\footnotetext{
${ }^{4}$ Here, we introduce the intermediary subscript $w$ to $e$ to remind us it is the weak basis.

${ }^{5}$ Again, the intermediary superscript $w$ is introduced for the neutrino.
}

quartic couplings. Since no beyond-the-SM scalars are found at the LHC, we make the conservative assumption that they are all heavier than $800 \mathrm{GeV}$. However, we are mindful that the optimal search strategy for a specific scalar is model dependent. Nevertheless, a robust prediction is that a universal suppression factor $U_{11}$ applies to all SM Higgs couplings which can be probed by the Higgs signal strengths at the LHC. The SM signal strength is parametrized by $\mu$ and is unity for the SM. The LHC- 1 bound is $\mu=1.09 \pm 0.11$ [15]. This implies $\left|U_{11}\right|^{2}>.87$ at $2 \sigma$ level. Hence, the mixing of $H_{0}$ with any of the other three scalars must be quite small or even vanishing. Small mixings can be achieved by tuning the couplings $\lambda_{2 \ell}, \lambda_{\Phi_{1} H_{0}}, \lambda_{\Phi_{2} H_{0}}$, and $\mu_{3}$.

\section{B. Two simplified cases of lepton mass diagonalization}

To capture the physics essence of this model, we will avoid the complication of keeping track of all the free parameters and focus on two simplified scenarios:

(i) Scenario A: We take $\lambda_{2 \ell}$ and $\mu_{3}$ to be small but finite, and we also assume that $Y_{2} \sim Y_{3} \sim Y$ and $\lambda_{1,2,3,4} \sim \bar{\lambda}$.

(ii) Scenario B: This is the limiting case of $\lambda_{1,2}=0=\mu_{3}$. We also assume that $Y_{2} \sim Y_{3} \sim Y$ and $\lambda_{3,4} \sim \bar{\lambda}$.

We shall refer to them as the Yukawa symmetry limits.

\section{Scenario A}

From Eq. (8), the charged lepton matrix in the basis $\mathcal{E}=$ $\left(e_{w}, E_{1}, E_{2}\right)$ is

$$
M_{E} \sim \frac{w}{\sqrt{2}}\left(\begin{array}{ccc}
y_{e} r & 0 & \bar{\lambda} \\
0 & Y r & \bar{\lambda} \\
\bar{\lambda} & \bar{\lambda} & Y r
\end{array}\right) .
$$

In the limit $r \rightarrow 0$ and $y, \bar{\lambda}$ finite, the charged lepton mass matrix has the structure

$$
\left(\begin{array}{lll}
0 & 0 & 1 \\
0 & 0 & 1 \\
1 & 1 & 0
\end{array}\right)
$$

The spectrum consists of a massless electron and two heavy degenerate leptons with mass $\sim \bar{\lambda} w$ in this limit. Returning to Eq. (12), it can be shown that the smallest eigenvalue is given by the larger of $y_{e}, Y$. This implies that in order to get the electron mass right, $Y \simeq y_{e}$. Thus, without loss of generality, we write the charged lepton mass matrix as

$$
M_{E} \simeq\left(\begin{array}{ccc}
m_{e} & 0 & \bar{\lambda} w / \sqrt{2} \\
0 & m_{e} & \bar{\lambda} w / \sqrt{2} \\
\bar{\lambda} w / \sqrt{2} & \bar{\lambda} w / \sqrt{2} & m_{e}
\end{array}\right),
$$

where $m_{e}$ is the physical electron mass. Moreover, the parameter $Y_{1}$ remains free. 
In general, we can write the physical mass eigenstates $\mathcal{E}_{\alpha}^{\prime}=\left(e, E_{-}, E_{+}\right)$, where $\alpha=1,2,3$ is given by

$$
\mathcal{E}_{i}^{\prime}=V_{i \alpha} \mathcal{E}_{\alpha}
$$

where $V$ is the unitary matrix that diagonalizes $M_{E}$ such that $\left(V^{A}\right)^{T} \cdot M_{E} \cdot V^{A}=\operatorname{diag}\left\{m_{e},-\left(\bar{\lambda} w-m_{e}\right), \bar{\lambda} w+m_{e}\right\}$. For the simplified symmetrical case of Eq. (14), $V^{A}$ can be worked out to be

$$
V^{A} \simeq\left(\begin{array}{ccc}
\frac{1}{\sqrt{2}} & \frac{1}{2} & \frac{1}{2} \\
-\frac{1}{\sqrt{2}} & \frac{1}{2} & \frac{1}{2} \\
0 & -\frac{1}{\sqrt{2}} & \frac{1}{\sqrt{2}}
\end{array}\right)
$$

The neutral lepton mass matrix is

$$
M_{N} \simeq \frac{\bar{\lambda} w}{\sqrt{2}}\left(\begin{array}{lll}
0 & 0 & 1 \\
0 & 0 & 1 \\
1 & 1 & 0
\end{array}\right) .
$$

Note that the difference between Eqs. (11) and (14) is proportional to an identical matrix. Therefore, both the neutral and charged lepton mass matrices are diagonalized by the same rotation, Eq. (16). At tree level, the spectrum consists of a massless neutrino and a Dirac neutrino of mass $\sim \bar{\lambda} w$. This can be seen by defining $n \mp=\frac{1}{\sqrt{2}}\left(\nu_{L}^{w} \mp N_{1 L}\right)$. In the basis $\left(n_{-}, n_{+}, N_{2 R}^{c}\right)$, the matrix $M_{N}$ becomes

$$
M_{N} \propto\left(\begin{array}{ccc}
0 & 0 & 0 \\
0 & 0 & 1 \\
0 & 1 & 0
\end{array}\right) .
$$

Clearly, $n_{-}$is massless, and the pair of Weyl neutrinos $n_{+}, N_{2 R}^{c}$ combines into a Dirac neutrino. In the case in which the neutrinos receive notable quantum corrections, we denote the charged neutral mass eigenstates as $\left(\nu, N_{-}, N_{+}\right)$with the convention $M_{N_{+}}>M_{N_{-}}$.

The $Y_{1}$ Yukawa term is relevant when considering the exotic fermion decays. In the mass basis, we have the following terms:

$$
\begin{aligned}
& \frac{Y_{1}}{2}\left\{-\bar{e}\left[\Re\left(H_{1}^{0}\right)+i \gamma_{5} \Im\left(H_{1}^{0}\right)\right] e+\frac{1}{\sqrt{2}} \bar{e}\left[\gamma_{5} \Re\left(H_{1}^{0}\right)+i \Im\left(H_{1}^{0}\right)\right]\right. \\
& \left.\times\left(E_{+}+E_{-}\right)+\frac{1}{2}\left(\bar{E}_{+}+\bar{E}_{-}\right)\left[\Re\left(H_{1}^{0}\right)+i \gamma_{5} \Im\left(H_{1}^{0}\right)\right]\left(E_{+}+E_{-}\right)\right\} \\
& -\frac{Y_{1}}{2}\left[\bar{\nu}+\left(\bar{N}_{+}+\bar{N}_{-}\right) / \sqrt{2}\right] \hat{R} e H_{1}^{+} \\
& +\frac{Y_{1}}{2 \sqrt{2}}\left[\bar{\nu}+\left(\bar{N}_{+}+\bar{N}_{-}\right) / \sqrt{2}\right] \hat{R}\left(E_{+}+E_{-}\right) H_{1}^{+}+\text {H.c. }
\end{aligned}
$$

\section{Scenario $B$}

In this case, the SM leptons completely decouple from the exotic fermion sector. The lepton matrices now become

$$
M_{E} \sim \frac{w}{\sqrt{2}}\left(\begin{array}{ccc}
y_{e} r & 0 & 0 \\
0 & Y r & \bar{\lambda} \\
0 & \bar{\lambda} & Y r
\end{array}\right),
$$

and $M_{E}$ can be diagonalized by the rotation matrix

$$
V^{B} \simeq\left(\begin{array}{ccc}
1 & 0 & 0 \\
0 & \frac{1}{\sqrt{2}} & \frac{1}{\sqrt{2}} \\
0 & -\frac{1}{\sqrt{2}} & \frac{1}{\sqrt{2}}
\end{array}\right) .
$$

The mass eigenstates are again denoted as $\left(e, E_{-}, E_{+}\right)$.

For neutrinos,

$$
M_{N}=\frac{w \lambda_{3}}{\sqrt{2}}\left(\begin{array}{ccc}
0 & 0 & 0 \\
0 & 0 & 1 \\
0 & 1 & 0
\end{array}\right) .
$$

Clearly, there is no mixing between the $\nu^{w}$ and $\left(N_{1 L}, N_{2 R}^{c}\right)$.

In the chiral basis, $M_{N}$ is also diagonalized by $V^{B}$, and the mass eigenstates are again denoted as $\left(\nu, N_{-}, N_{+}\right)$. At tree level, $N_{+}, N_{-}$are degenerated. In fact, $N_{1 L}$ and $N_{2 R}$ form a Dirac fermion at tree level (let us simply call it $N$ ), and $\hat{L} N=N_{1 L}, \hat{R} N=N_{2 R}$. The degeneracy will be broken by the one-loop mass correction. However, the quantum correction is expected to be much smaller than $w$, and taking $N$ as a Dirac DM is a good approximation.

The $Y_{1}$ Yukawa term in the mass basis becomes

$$
\frac{Y_{1}}{\sqrt{2}}\left(\bar{\nu} H_{1}^{+}+\bar{e} H_{1}^{0}\right) \hat{R}\left(E_{+}+E_{-}\right)+\text {H.c. }
$$

The heavier of $E_{ \pm}$and $H_{1}$ can decay via this Yukawa interaction followed by the lighter one decaying through gauge interactions.

\section{GAUGE INTERACTIONS}

The covariant derivative is

$$
D_{\mu}=\partial_{\mu}-i \frac{g}{2} \mathbf{W}_{\mu} \cdot \tau-i g^{\prime} Y B_{\mu}-i g_{\mathfrak{l}}(\ell) Z_{\mathfrak{l} \mu},
$$

where $Z_{\mathfrak{l}}$ is the gauge boson for $U(1)_{\mathfrak{l}}, Y$ is the hypercharge, and $\ell$ is the lepton number. All the quantum numbers can be read from the tables. Other notations are standard. After the SSB of $U(1)_{\mathfrak{l}},\left\langle\Phi_{1,2}\right\rangle=w_{1,2}$, the $Z_{\mathfrak{l}}$ acquires a mass, 


$$
\begin{aligned}
M_{X} & =g_{\mathfrak{l}} \sqrt{w_{1}^{2}+4 w_{2}^{2}}=g_{\mathfrak{l}} \bar{w} \\
& =2.24 g_{\mathfrak{l}} w \quad \text { for } w_{1}=w_{2}=w,
\end{aligned}
$$

where $\bar{w}^{2}=w_{1}^{2}+4 w_{2}^{2}$ gives the overall lepton numberviolating scale.

In terms of the physical gauge bosons, the gauge interaction in the weak basis is

$$
\begin{aligned}
& i e\left[\bar{e}_{w} \gamma^{\mu} e_{w}+\bar{E}_{1} \gamma^{\mu} E_{1}+\bar{E}_{2} \gamma^{\mu} E_{2}\right] P_{\mu} \\
& \quad-\frac{i g_{2}}{2 c_{w}}\left[\bar{\nu}_{w} \gamma^{\mu} \hat{L} \nu_{w}+\bar{N}_{1} \gamma^{\mu} \hat{L} N_{1}+\bar{N}_{2} \gamma^{\mu} \hat{R} N_{2}\right] Z_{\mu} \\
& \quad-\frac{i g_{2}}{c_{w}}\left[\bar{e}_{w} \gamma^{\mu}\left(g_{L} \hat{L}+g_{R} \hat{R}\right) e_{w}+\bar{E}_{1} \gamma^{\mu}\left(g_{L} \hat{L}+g_{R} \hat{R}\right) E_{1}\right. \\
& \left.\quad+\bar{E}_{2} \gamma^{\mu}\left(g_{L} \hat{R}+g_{R} \hat{L}\right) E_{2}\right] Z_{\mu} \\
& -\frac{i g_{2}}{\sqrt{2}}\left[\bar{\nu}_{w} \gamma^{\mu} \hat{L} e_{w}+\bar{N}_{1} \gamma^{\mu} \hat{L} E_{1}+\bar{N}_{2} \gamma^{\mu} \hat{R} E_{2}\right] W_{\mu}^{+}+\text {H.c. } \\
& -i g_{\mathfrak{l}}\left[\bar{\nu}_{w} \gamma^{\mu} \hat{L} \nu_{w}+\bar{e}_{w} \gamma^{\mu} e_{w}-\bar{N}_{1} \gamma^{\mu} \hat{L} N_{1}-\bar{E}_{1} \gamma^{\mu} E_{1}\right]\left(Z_{\mathfrak{l}}\right)_{\mu},
\end{aligned}
$$

where $P$ denotes the photon and $g_{L}=-1 / 2+s_{w}^{2}$ and $g_{R}=$ $s_{w}^{2}$ are the SM left-handed and right-handed $Z$-electron couplings, respectively. We have also assumed the kinetic mixing between $U(1)_{\mathfrak{l}}$ and $U(1)_{Y}$ is negligible.

\section{A. SM gauge interaction}

For the scenario B, the weak basis and the mass basis are related by

$$
\begin{gathered}
e_{w}=e, \\
E_{1}=\frac{1}{\sqrt{2}}\left[E_{+}+E_{-}\right], \\
E_{2}=\frac{1}{\sqrt{2}}\left[E_{+}-E_{-}\right], \\
\nu_{w}=\nu, \\
N_{1 L}=\hat{L} N=\frac{1}{\sqrt{2}}\left[N_{+}+N_{-}\right], \\
N_{2 R}=\hat{R} N=\frac{1}{\sqrt{2}}\left[N_{+}^{c}-N_{-}^{c}\right] .
\end{gathered}
$$

The mass splitting between $N_{+}$and $N_{-}$could stem from the quantum corrections and is unlikely to be experimentally detectable. Hence, it is a very good approximation to lump them into a Dirac fermion $N$.

The QED interaction in the mass basis remains intact,

$$
i e\left[\bar{e} \gamma^{\mu} e+\bar{E}_{+} \gamma^{\mu} E_{+}+\bar{E}_{-} \gamma^{\mu} E_{-}\right] P_{\mu} .
$$

The SM-charged current interaction becomes

$$
-\frac{i g_{2}}{\sqrt{2}}\left[\bar{\nu} \gamma^{\mu} \hat{L} e+\frac{1}{\sqrt{2}} \bar{N} \gamma^{\mu} E_{+}-\frac{1}{\sqrt{2}} \bar{N} \gamma^{\mu} \gamma^{5} E_{-}\right] W_{\mu}^{+}+\text {H.c. }
$$

The SM-charged current interaction is intact. However, note that the $\bar{N} E_{+} W^{+}$vertex is vectorlike and the $\bar{N} E_{-} W^{+}$ one is axial vector.

The SM-neutral current interaction admits a similar structure and becomes

$$
\begin{aligned}
\{- & \frac{i g_{2}}{2 c_{w}}\left[\bar{\nu} \gamma^{\mu} \hat{L} \nu+\bar{N} \gamma^{\mu} N\right]-\frac{i g_{2}}{c_{w}}\left[\bar{e} \gamma^{\mu}\left(g_{L} \hat{L}+g_{R} \hat{R}\right) e\right] \\
& -\frac{i g_{2}}{c_{w}} \frac{g_{L}+g_{R}}{2}\left[\bar{E}_{+} \gamma^{\mu} E_{+}+\bar{E}_{-} \gamma^{\mu} E_{-}\right] \\
& \left.-\frac{i g_{2}}{c_{w}} \frac{g_{R}-g_{L}}{2}\left[\bar{E}_{+} \gamma^{\mu} \gamma^{5} E_{-}+\bar{E}_{-} \gamma^{\mu} \gamma^{5} E_{+}\right]\right\} \times Z_{\mu} .
\end{aligned}
$$

For scenario A,

$$
\begin{aligned}
e_{w} & =\frac{e}{\sqrt{2}}+\frac{1}{2}\left[E_{+}+E_{-}\right], \\
E_{1} & =-\frac{e}{\sqrt{2}}+\frac{1}{2}\left[E_{+}+E_{-}\right], \\
E_{2} & =\frac{1}{\sqrt{2}}\left[E_{+}-E_{-}\right], \\
\nu_{L}^{w} & =\frac{\nu}{\sqrt{2}}+\frac{1}{2}\left[N_{+}+N_{-}\right], \\
N_{1 L} & =-\frac{\nu}{\sqrt{2}}+\frac{1}{2}\left[N_{+}+N_{-}\right], \\
N_{2 R} & =\frac{1}{\sqrt{2}}\left[N_{+}^{c}-N_{-}^{c}\right] .
\end{aligned}
$$

Again, we adopt the approximation in which $N_{+}, N_{-}$form a Dirac fermion $N$. The neutrinos in the interaction basis become

$\nu_{L}^{w} \simeq \frac{1}{\sqrt{2}}(\nu+\hat{L} N), \quad N_{1 L} \simeq \frac{1}{\sqrt{2}}(-\nu+\hat{L} N), \quad N_{2 R} \simeq \hat{R} N$.

And it is easy to check that the QED, SM-charged current, and SM-neutral current parts are the same as those in scenario B.

In these two cases we discussed, the SM gauge couplings are intact. This is due to the fact that $V_{31}=0$ for both cases. In general, the SM Z-e-e and $Z-\nu-\nu$ axial-vector part couplings can deviate from the SM prediction. However, the deviation is expected to be small, which is controlled by the mixing, $\sim \mathcal{O}\left(m_{l} / v_{1}\right)<10^{-7}$, between the SM lepton and $L_{2}$. 


\section{B. $Z_{\mathfrak{Y}}$ interactions}

The corrections from any extra $Z$-boson couplings to SM leptons are important for low-energy high-sensitivity experiments, which can be done at the proposed lepton colliders such as the international linear collider (ILC) [16] and compact linear collider (CLIC) [17]. And it is particularly true for $Z_{\mathfrak{r}}$. To facilitate such studies, we need to know the couplings of $Z_{\mathfrak{l}}$ to the SM leptons, which are also important for direct searches.

We begin with the couplings of charged leptons. It is easy to see from Eq. (24) and Table I that the charged leptons $\mathcal{E}=\left(e_{w}, E_{1}, E_{2}\right)$ have vector couplings to $Z_{\mathfrak{l}}$ in the gauge basis. We define a charged matrix $Q^{\mathcal{E}}$ representing this coupling by $\overline{\mathcal{E}} Q^{\mathcal{E}} \gamma_{\mu} \mathcal{E} Z_{\mathfrak{l}}^{\mu}$, where

$$
Q^{\mathcal{E}}=\left(\begin{array}{ccc}
1 & 0 & 0 \\
0 & -1 & 0 \\
0 & 0 & 0
\end{array}\right) \text {. }
$$

In the mass basis, the corresponding charge mass is given by

$$
Q^{\mathcal{E}}=V^{\dagger} Q^{\mathcal{E}} V,
$$

where $V$ is given by Eq. (15). Specific examples of $V$ are given in Eqs. (16) and (21). In general, $Q^{\prime \mathcal{E}}$ is not even a diagonal matrix, and this is in sharp contrast to the case of the SM gauge interactions. Of particular interest is $Q_{11}^{\mathcal{E}}=\left|V_{e 1}\right|^{2}-\left|V_{E 1}\right|^{2}$, which determines the coupling strength of $Z_{\mathfrak{l}}$ to the physical electrons. For scenario A, we see that not only is this suppressed by matrix elements but that an accidental cancelation also occurs. Indeed, for the simplified case, it vanishes as from Eq. (16).

Similarly, we find $Q_{12}^{\mathcal{E}}=V_{e 1}^{*} V_{e 2}-V_{E 1}^{*} V_{E 2}$, which gives the off-diagonal coupling of $Z_{\mathfrak{l}}$ to the physical electron and new heavy charged lepton. Explicitly, we have for scenario A and in the Yukawa symmetry limit

$$
-\frac{i g_{\mathfrak{l}}}{\sqrt{2}}\left[\bar{e} \gamma^{\mu}\left(E_{+}+E_{-}\right)+\left(\bar{E}_{+}+\bar{E}_{-}\right) \gamma^{\mu} e\right] Z_{\mathfrak{l} \mu} .
$$

In general, the coupling $Q_{11}^{\mathcal{E}} \bar{e} \gamma_{\mu} e Z_{\mathfrak{l}}^{\mu}$ will not vanish since the $\lambda$ 's are all different and a complete cancelation is not expected. Moreover, it is expected that $\left|Q^{\mathcal{E}}\right|<1$.

On the other hand, it is very different for scenario B. In this case, the SM electron decouples from the exotic fermion, and one has $Q_{11}^{\mathcal{E}}=1$. Instead, we have

$$
i g_{\mathfrak{l}}\left[\frac{1}{2}\left(\bar{E}_{+}+\bar{E}_{-}\right) \gamma^{\mu}\left(E_{+}+E_{-}\right)-\bar{e} \gamma^{\mu} e\right] Z_{\mathfrak{l} \mu} .
$$

In both scenarios, the couplings are vectorial.

Similar considerations for the neutral leptons give for scenario A

$$
-\frac{i g_{\mathfrak{l}}}{2}\left[\bar{\nu} \gamma^{\mu} \hat{L} N+\bar{N} \gamma^{\mu} \hat{L} \nu\right] Z_{\mathfrak{l} \mu}
$$

and for scenario $\mathrm{B}$

$$
i g_{\mathfrak{l}}\left[\bar{N} \gamma^{\mu} \hat{L} N-\bar{\nu} \gamma^{\mu} \hat{L} \nu\right] Z_{\mathfrak{l} \mu} .
$$

In contrast to the charged leptons, these couplings are left handed.

\section{PHENOMENOLOGY OF $Z_{\mathfrak{l}}$}

It is clear that $Z_{\mathfrak{l}}$ has only tree-level couplings to $\mathrm{SM}$ leptons and not to quarks. Hence, its phenomenology is very different from most extra $Z$ extensions of the SM.

For scenario $A$ in the Yukawa symmetry limit, $Z_{\mathfrak{l}}$ does not couple to SM charged leptons phenomenology at tree level, although the one-loop effect can exist. Thus, we do not expect such probes to be sensitive to $Z_{\mathfrak{l}}$ in this limiting case. On the other hand, for scenario B, this coupling is at full strength. Therefore, in the following, we mainly focus on the $Z_{l}$ phenomenology for scenario $\mathrm{B}$. In between the two cases, our results can be used by properly multiplying by the appropriate factor $\left(Q^{\prime \mathcal{E}}\right)^{2}$ once elements of the mixing matrix $V$ are determined.

The model has many parameters. However, most of them are related to the exotic scalars. For $Z_{\mathfrak{l}}$ phenomenology, they largely do not play a role. The controlling parameters are $g_{\mathfrak{l}}$ and $M_{X}$, the mass of $Z_{\mathfrak{l}}$. Whether the new leptons and scalars are heavier or lighter than $Z_{\mathfrak{l}}$ mainly affects the branching ratio of $Z_{\mathfrak{l}}$ into SM states and is of secondary importance here. For definiteness, we shall assume that $Z_{\mathfrak{l}}$ is the lightest of the new particles.

Direct production from $e^{+} e^{-}$colliders via $e^{+} e^{-} \rightarrow Z_{\mathfrak{l}}$, which subsequently decays into $\ell^{+} \ell^{-}$pairs, gives an unambiguous signal if kinematically allowed. Indirect virtual exchange of $Z_{\mathfrak{l}}$ effects can be discerned in lowenergy precision experiments involving only leptons. Some notable reactions are studied below.

\section{A. LEP II bound}

The four-lepton contact interactions between electrons and charged leptons $\ell$ with scale $\Lambda_{V V}{ }^{6}$ are parametrized by

$$
\frac{4 \pi}{\left(\Lambda_{V V}\right)^{2}}\left(\bar{e} \gamma^{\mu} e\right)\left(\bar{l} \gamma_{\mu} l\right)
$$

This can be generated by exchanging a heavy $Z_{\mathfrak{l}}$ boson with the coupling $g_{\mathfrak{l}}$. Since the leptons are mass eigenstates, the coupling has to be scaled by the factor $Q_{11}^{\mathcal{E}}$. The operator yields a destructive interference with the SM process for $\sqrt{s} \lll M_{X}$. The effects of the contact

\footnotetext{
${ }^{6}$ Note that if $l=e$ there will be an extra symmetry factor 2 in the denominator of Eq. (41).
} 
interactions have been searched for at the large electronpositron collider (LEP). A limit $\Lambda_{V V}>20.0 \mathrm{TeV}$ is set if the universality between leptons is assumed [18]. This amounts to a $\rho$-dependent lower bound on $M_{X}$,

$$
M_{X} \geq \sqrt{\rho} \sqrt{\alpha} \times 20.0 \mathrm{TeV} \sim 1.77 \sqrt{\rho} \mathrm{TeV},
$$

where $\rho \equiv\left(g_{\mathfrak{l}} / e\right)^{2}$. For example, $M_{X}>0.97 \mathrm{TeV}$ if $\rho=0.3$. The above limit works for scenario B, in which $Q_{11}^{\mathcal{E}}=1$. On the other hand, there is no such tree-level contact interaction for scenario A since $Q_{11}^{\mathcal{E}}=0$ and the LEP bound does not apply at all.

For the remainder of this section, scenario B is assumed.

\section{B. $Z_{\mathfrak{l}}$ width}

With the assumptions listed, the main decay modes of $Z_{\mathfrak{l}}$ are into the SM leptons. The total width can be calculated to be

$$
\Gamma_{Z_{\mathfrak{l}}}=\sum_{l} \frac{\alpha \rho}{6} M_{X}\left(1+2 x_{l}\right) \sqrt{1-4 x_{l}}\left[\left(l_{L}^{l}\right)^{2}+\left(l_{R}^{l}\right)^{2}\right],
$$

where $x_{l}=\left(m_{l} / M_{X}\right)^{2}, \alpha$ is the fine structure constant, and $l_{L, R}^{l}$ is the left-/right-handed coupling for the $l$ lepton flavor. Since $x_{l} \ll 1$, we have $\Gamma_{Z_{\mathrm{l}}}=\frac{3}{2} \alpha \rho M_{X}$. For a light $Z_{\mathfrak{l}}$, $M \sim \mathcal{O}(100) \mathrm{GeV}$, its typical width is around a few $\mathrm{GeV}$, and its decay branching ratios are $\operatorname{Br}\left(Z_{\mathfrak{l}} \rightarrow l^{+} l^{-}\right)=$ $2 / 9$ and $\operatorname{Br}\left(Z_{\mathfrak{l}} \rightarrow \nu \bar{\nu}\right)=1 / 9$ for each flavor.

\section{Front-back asymmetry $\left(A_{F B}\right)$ in $e^{+} e^{-} \rightarrow \mu^{+} \mu^{-}$}

The exchange of $Z_{\mathfrak{l}}$, which has vector couplings to $e, \mu$, will interfere with the SM exchange of $Z, \gamma$. The differential cross section is given by

$$
\begin{aligned}
\frac{d \sigma}{d \cos \theta}= & \frac{\pi \alpha^{2}}{2 s}\left\{\left|D_{\gamma \mathfrak{l}}\right|^{2}\left(1+\cos ^{2} \theta\right)\right. \\
& +\frac{1}{4\left(s_{W} c_{W}\right)^{4}}\left|D_{Z}\right|^{2}\left[\left(g_{L}^{2}+g_{R}^{2}\right)^{2}\left(1+\cos ^{2} \theta\right)\right. \\
& \left.+2\left(g_{L}^{2}-g_{R}^{2}\right)^{2} \cos \theta\right] \\
& +\frac{1}{2\left(s_{W} c_{W}\right)^{2}} \Re\left(D_{\gamma \mathfrak{l}}^{*} D_{Z}\right)\left[\left(g_{L}+g_{R}\right)^{2}\left(1+\cos ^{2} \theta\right)\right. \\
& \left.\left.+2\left(g_{L}-g_{R}\right)^{2} \cos \theta\right]\right\},
\end{aligned}
$$

where $\theta$ is the scattering angle of $\mu^{-}, s$ is the center-of-mass energy squared, and $c_{W}\left(s_{W}\right)$ is the cosine (sine) of the weak mixing angle. Also, the SM Z -lepton couplings are $g_{L}=-\frac{1}{2}+s_{W}^{2}$ and $g_{R}=s_{W}^{2}$. We have also introduced the dimensionless gauge boson propagator factors,

$$
\begin{aligned}
D_{\gamma \mathfrak{l}} & =1+\frac{\rho s}{s-M_{X}^{2}+i M_{X} \Gamma_{X}}, \\
D_{Z} & =\frac{s}{s-M_{Z}^{2}+i M_{Z} \Gamma_{Z}},
\end{aligned}
$$

where $M_{X}$ is the mass and $\Gamma_{X}$ is the width of $Z_{l}$. We have combined the photon and $Z_{\mathfrak{l}}$ exchange together since both have vector couplings to $e$ and $\mu$. The finite widths are included to take care of the behaviors near the mass poles. The SM $\gamma-Z$ interference causes a wiggling with magnitude around $\sim \mathcal{O}\left(10^{-2}\right)$ of the cross section around the $Z$ pole; see Figs. 1(a) and 1(b). This along with other asymmetries has been experimentally confirmed by analyzing the $Z$-line shape $[18,19]$. The presence of the new $Z_{\mathfrak{l}}$ boson provides additional wiggling around the SM $Z$ pole at the level of $\sim \mathcal{O}\left(10^{-3}\right)$, Fig. 1(b). This will be the first unambiguous sign of the existence of a new gauge boson which interferes with $\gamma$, $Z$, which can be searched for at the future $Z$ factories such as the $e^{+} e^{-}$collisions at the future circular collider [20] and circular electron positron collider [21].

We parametrize the cross section as

$$
\frac{d \sigma}{d \cos \theta}=\frac{\pi \alpha^{2}}{2 s}\left[A\left(1+\cos ^{2} \theta\right)+B \cos \theta\right] .
$$

Then, $A_{F B}$ is given by

$$
A_{F B}=\frac{\int_{0}^{1} d \cos \theta \frac{d \sigma}{d \cos \theta}-\int_{-1}^{0} d \cos \theta \frac{d \sigma}{d \cos \theta}}{\int_{-1}^{1} d \cos \theta \frac{d \sigma}{d \cos \theta}}=\frac{3 B}{8 A},
$$

where $A, B$ can be easily read from Eq. (44). $A_{F B}$ is centerof-mass energy dependent.

At the $Z$ pole, we have

$$
A_{F B}=\frac{3}{4} \frac{\left(g_{R}^{2}-g_{L}^{2}\right)^{2}}{\left(g_{R}^{2}+g_{L}^{2}\right)^{2}} \sim 0.01695
$$

by using $s_{W}^{2}=0.2311$. It is accidentally small because $s_{W}^{2}$ is very close to $1 / 4$. It is interesting to note that the $Z_{\mathfrak{l}}$

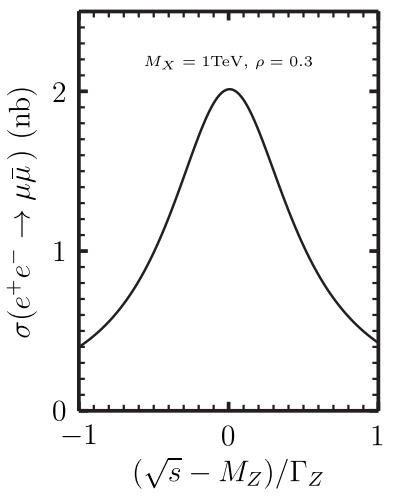

(a)

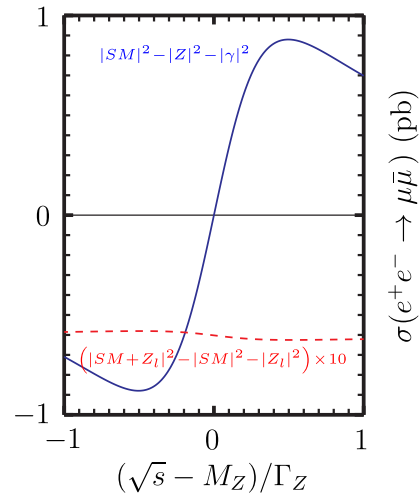

(b)
FIG. 1. (a) The line shape of the $e^{+} e^{-} \rightarrow \mu \bar{\mu}$ cross section near the $Z$ pole for $M_{X}=1 \mathrm{TeV}$ and $\rho=0.3$. (b) The SM photon- $Z$ interference and the $Z_{l}$-SM interference. 
exchange induces a universal positive contribution to all $A_{F B}^{l}$ for SM charged leptons at the $Z$ pole. This can be understood as follows. First, as $Z_{\mathfrak{l}}$ is heavier than $M_{Z}$, it gives a destructive interference to the symmetric $D_{\gamma l}$ term and reduces $A$. Second, the asymmetric $\left(g_{L}-g_{R}\right)^{2}$ $\left(\gg\left(g_{L}^{2}-g_{R}^{2}\right)^{2}\right)$ term from $Z_{l}$-SM interference increases $B$ in Eq. (47). On the other hand, the $A_{F B}^{q}$ for SM quarks receive no such contributions. Therefore, with the presence of $Z_{\mathfrak{l}}$ and $M_{X}>M_{Z}, \tilde{A}_{F B}^{l}>\tilde{A}_{F B}^{q}$, where $\tilde{A}_{F B} \equiv A_{F B} / A_{F B}^{S M}$ is a robust prediction. For $A_{F B}^{b}$, the LEP experimental value is measured to be $0.0992(16)$, and the SM expectation is 0.1031 (3) [22] by using the above value of $s_{W}$ and all other SM parameters input from the global electroweak precision fit, or roughly speaking, $\tilde{A}_{F B}^{l} / \tilde{A}_{F B}^{b}=1.0393 \pm 0.0164$.

However, taking into account the mass bound, Eq. (42), only a $10.7 \mathrm{TeV} Z_{\mathfrak{l}}$ with $\rho \sim 36.3$ can explain this difference between the lepton and quark sector at $2 \sigma$ level. In other words, with $g_{\mathfrak{l}} \sim 6 e$, one can account for this discrepancy. Certainly, whether this difference is due to $Z_{l}$ will be clarified at a future $Z$-factory option $e^{+} e^{-}$ colliders.

Beyond the $Z$ pole and for $M_{Z} \ll \sqrt{s} \ll M_{X}$,

$$
\begin{aligned}
A_{F B} & \sim \frac{3}{4} \frac{\left(g_{R}^{2}-g_{L}^{2}\right)^{2}+2\left(s_{W} c_{W}\right)^{2}\left(g_{R}-g_{L}\right)^{2}}{4\left(s_{W} c_{W}\right)^{4}+\left(g_{R}^{2}+g_{L}^{2}\right)^{2}+2\left(s_{W} c_{W}\right)^{2}\left(g_{R}+g_{L}\right)^{2}} \\
& \sim 0.4691 .
\end{aligned}
$$

At the $Z_{\mathfrak{l}}$ pole, the asymmetry is small due to the vector coupling nature of $Z_{\mathfrak{l}}$, and it becomes

$$
\begin{aligned}
A_{F B} & \sim \frac{3}{4} \frac{\left(g_{R}-g_{L}\right)^{2}}{\left(g_{R}+g_{L}\right)^{2}+2\left(s_{W} c_{W}\right)^{2} \frac{2}{3 \alpha}} \\
& \sim 8.83 \times 10^{-6}
\end{aligned}
$$

by using Eq. (43). It is interesting that the above three values are not sensitive to $\rho$. Finally, for $\sqrt{s} \gg M_{X}$,

${ }_{3}^{4} A_{F B} \sim \frac{\left(g_{R}^{2}-g_{L}^{2}\right)^{2}+2 \bar{\rho}\left(s_{W} c_{W}\right)^{2}\left(g_{R}-g_{L}\right)^{2}}{4\left(s_{W} c_{W}\right)^{4} \bar{\rho}^{2}+\left(g_{R}^{2}+g_{L}^{2}\right)^{2}+2 \bar{\rho}\left(s_{W} c_{W}\right)^{2}\left(g_{R}+g_{L}\right)^{2}}$,

where $\bar{\rho}=1+\rho$. We give a plot of $A_{F B}$, Fig. 2 , for $M_{X}=$ $2 \mathrm{TeV}$ and $\rho=0.3,1.0$.

The $\sqrt{s}$-dependent $A_{F B}$ provides an important handle to probe the new heavy gauge boson. It is especially useful for the planned linear colliders. For example, the CLIC has plans for two-staged intermediate energy at $\sqrt{s}=$ $0.5,1.4(1.5) \mathrm{TeV}$ before reaching its ultimate $3 \mathrm{TeV}$ goal [17]. At each new stage, it is able to tune down the energy by about a factor 3 without losing the luminosity. One might be able to see the effect of the new gauge boson in the $A_{F B}$ even if $Z_{\ell}$ is too heavy to be produced on shell.

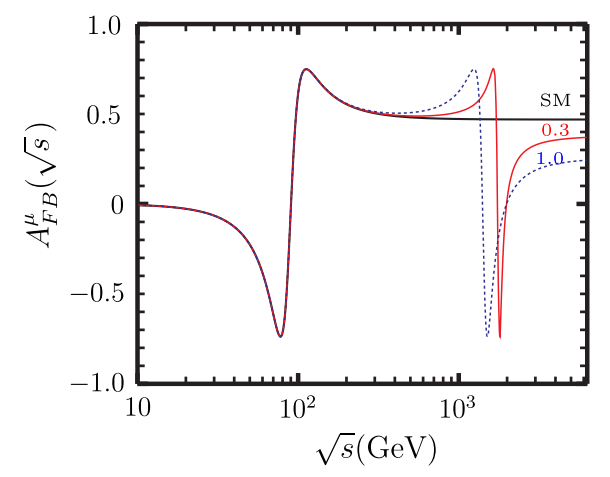

FIG. 2. The $A_{F B}$ vs $\sqrt{s}$ with $M_{X}=2 \mathrm{TeV}$. The black curve is for the SM, and the red (blue) curve is for a $Z_{\mathfrak{l}}$ with $\rho=0.3$ (1.0).

\section{Muon $g-2$}

The $Z_{\mathfrak{l}}$ contribution to the muon anomalous moment can be easily calculated to be

$$
\Delta a_{\mu}=\frac{\alpha}{3 \pi} \rho\left(\frac{m_{\mu}^{2}}{M_{X}^{2}}\right) .
$$

Using the value $a_{\mu}^{\exp }-a_{\mu}^{\text {th }}=2.88 \times 10^{-9}$ [22] and requiring that $\Delta a_{\mu}$ is smaller than that, we obtain the constraint $M_{X}>54.5 \sqrt{\rho} \mathrm{GeV}$. The helicity flip factor severely curtails the sensitivity of $a_{\mu}$ to $M_{X}$. The new exotic scalars have contributions to $\Delta a_{\mu}$ as well. Since their masses have to be heavier than $\sim 0.8 \mathrm{TeV}$, those contributions are negligible. However, this limit cannot compete with Eq. (42). For a recent review of the connection between $a_{\mu}$ and the new physics, see Ref. [23].

\section{E. Møeller scattering}

The exchange of $Z_{\mathfrak{l}}$ will interfere with the SM $Z, \gamma$ processes at the amplitude level. The leading order is free of hadronic uncertainties and hence offers a very clean sensitive probe of $Z_{\mathfrak{l}}$. Since the $Z_{\mathfrak{l}}$ admits vector coupling to the electron, it does not contribute left-right asymmetry directly. Its role in the Mø eller scattering is to increase the symmetric cross section from the photon exchange diagram. The asymmetry is then reduced to

$$
\begin{gathered}
A_{L R} \simeq A_{L R}^{\mathrm{SM}} \times\left[1-6 \frac{\rho Q^{2}}{M_{X}^{2}} \frac{(1-y)\left(1-y+y^{2}\right)}{1+y^{4}+(1-y)^{4}}\right], \\
A_{L R}^{\mathrm{SM}}=\frac{4 G_{\mu} s}{\sqrt{2} \pi \alpha} \frac{y(1-y)}{1+y^{4}+(1-y)^{4}}\left[\frac{1}{4}-s_{w}^{2}\right],
\end{gathered}
$$

where $y=-\frac{t}{s}$. The asymmetry was measured to be

$$
A_{L R}=131 \pm 14(\text { stas }) \pm 10 \text { (syst) ppb }
$$

by the SLAC E158 experiment [24], where $Q^{2}=$ $0.026(\mathrm{GeV})^{2}, \quad y=0.6$; thus, $A_{L R}^{\mathrm{SM}}=1.47 \times 10^{-7}$. By 

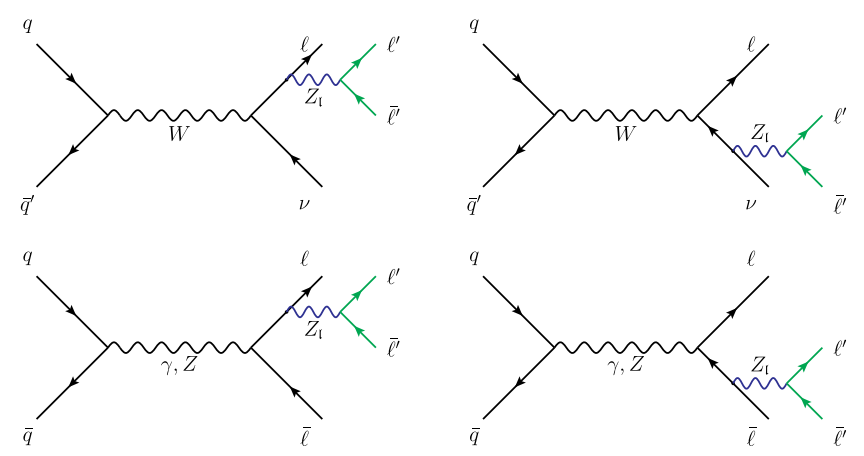

FIG. 3. Drell-Yan production of $Z_{\mathfrak{l}}$.

taking the 95\% C.L. limit, $-0.337<\frac{\delta A_{L R}}{A_{L R}}<0.122$. Because of the stringent limits from Eq. (42), the $Z_{\mathfrak{l}}$ contribution to $\frac{\delta A_{L R}}{A_{L R}}$ has no significance at all.

\section{F. $Z_{\mathfrak{l}}$ production at the $\mathrm{LHC}$}

If energetically allowed, $Z_{\mathfrak{l}}$ can be produced at the LHC via the radiaitive Drell-Yan process as depicted in Fig. 3.

The final states will be two pairs of leptons with different flavors in which one pair constitutes a resonance, e.g., $\mu^{+} \mu^{-}$and $e^{+} e^{-}$pairs and either pair coming from on-shell $Z_{\mathfrak{l}}$ decay. Another signal will be three leptons plus missing energy. A spectacular example will be a $\mu^{+} \mu^{-}$and an $e^{+} e^{-}$ pair with either pair resulting from $Z_{\mathfrak{l}}$ decay. Neither signature will have any jet activities.

Here, just for illustration purposes, we consider the signal $p p \rightarrow e^{+} e^{-} Z_{\mathfrak{l}} \rightarrow e^{+} e^{-}+(\mu \bar{\mu})$, and there is a sharp resonance peak of muon pair invariant mass at $M_{X}$. The SM background is $p p \rightarrow e^{+} e^{-} \mu^{+} \mu^{-}$with $m_{\mu \bar{\mu}} \sim M_{X}$. We evaluate the cross section of $p p \rightarrow e^{+} e^{-} Z_{\mathfrak{l}}$ at the LHC for three c.m. energies with the program CALCHEP [25] with the CTEQ6L1 parton distribution functions set [26]. The standard model background are also evaluated with CALCHEP with a cut that $m_{\mu \bar{\mu}} \in\left(M_{X}-50 \mathrm{GeV}, M_{X}+50 \mathrm{GeV}\right)$. The numbers are listed in Table III.

We use $S / \sqrt{B}=3$ and an integrated luminosity $\mathcal{L}_{0}=$ $3000(f b)^{-1}$ as the benchmark limit of detecting a $Z_{l}$ at the LHC. Then,

$$
\begin{aligned}
3 & =\frac{\sigma\left(p p \rightarrow e^{+} e^{-} Z_{\mathfrak{l}}(\mu \bar{\mu})\right) \operatorname{Br}\left(Z_{\mathfrak{l}} \rightarrow \mu \bar{\mu}\right) \times \mathcal{L}_{0}}{\sqrt{\sigma_{B G} \times \mathcal{L}_{0}}} \\
& =\frac{2}{9} \sqrt{\frac{\mathcal{L}_{0}}{\sigma_{B G}}}\left(\frac{\sigma}{g_{\mathfrak{l}}^{2}}\right) g_{\mathfrak{l}}^{2} .
\end{aligned}
$$

The corresponding highest lepton number-breaking scale we can probe is $\bar{w}_{\max }^{2}=(2 / 27) \sqrt{\mathcal{L}_{0} / \sigma_{B G}} \times$ $\left(\sigma / g_{\mathfrak{l}}^{2}\right) M_{X}^{2}$, which is also displayed in Table III. The LHC14, LHC30, and LHC100 have the potential to probe the lepton number-violating scales up to $\sim 0.5,1.0$, and $2.0 \mathrm{TeV}$, respectively.
TABLE III. The $p p \rightarrow e^{+} e^{-} Z_{l}$ cross section normalized by $g_{l}^{2}$ and the SM background (BG). The cross sections are in $(p b)$, and $\bar{w}_{\max }$ are in TeV.

\begin{tabular}{cccccc}
\hline \hline$\frac{\sqrt{s}}{\mathrm{TeV}}$ & & $\frac{M_{X}}{\mathrm{TeV}}=0.5$ & $\frac{M_{X}}{\mathrm{TeV}}=1.0$ & $\frac{M_{X}}{\mathrm{TeV}}=2.0$ & $\frac{M_{X}}{\mathrm{TeV}}=5.0$ \\
\hline 14 & $\frac{\sigma}{g_{l}^{2}}$ & $5.4 \times 10^{-5}$ & $1.7 \times 10^{-6}$ & $1.9 \times 10^{-8}$ & $9.8 \times 10^{-13}$ \\
& $\sigma_{B G}$ & $2.2 \times 10^{-5}$ & $1.4 \times 10^{-6}$ & $5.4 \times 10^{-8}$ & $6.2 \times 10^{-11}$ \\
& $\frac{\bar{w}_{\max }}{\mathrm{TeV}}$ & 0.61 & 0.43 & $\ldots$ & $\ldots$ \\
30 & $\frac{\sigma}{g_{l}^{2}}$ & $2.6 \times 10^{-4}$ & $1.5 \times 10^{-5}$ & $5.1 \times 10^{-7}$ & $1.0 \times 10^{-9}$ \\
& $\sigma_{B G}$ & $6.8 \times 10^{-5}$ & $7.1 \times 10^{-6}$ & $4.2 \times 10^{-7}$ & $5.7 \times 10^{-9}$ \\
& $\frac{\bar{w}_{\max }}{\mathrm{TeV}}$ & 1.02 & 0.85 & $\ldots$ & $\ldots$ \\
100 & $\frac{\sigma}{g_{l}^{2}}$ & $1.7 \times 10^{-3}$ & $1.5 \times 10^{-4}$ & $1.1 \times 10^{-5}$ & $1.8 \times 10^{-7}$ \\
& $\sigma_{B G}$ & $3.0 \times 10^{-4}$ & $3.2 \times 10^{-5}$ & $2.8 \times 10^{-6}$ & $7.6 \times 10^{-8}$ \\
& $\frac{\bar{w}_{\max }}{\mathrm{TeV}}$ & 1.79 & 1.85 & 1.83 & $\ldots$ \\
\hline \hline
\end{tabular}

\section{RADIATIVE SEESAW MASS FOR $\nu_{L}$}

The Feynman diagrams for radiative $\nu_{L}$ mass generation are depicted in Fig. 4 and are given in the weak eigenbasis. They fill in the upper left-hand block of zeros in Eq. (18). ${ }^{7}$

In the limit at which the charged $H_{1}, S$ scalars are heavier than the leptons, we get

$$
M_{11}=\frac{f\left(Y_{1} v\right)\left(\lambda_{1 \ell} w\right) m_{e}}{16 \pi^{2}\left(m_{1}^{2}-m_{S}^{2}\right)} \ln \left(\frac{m_{1}^{2}}{m_{S}^{2}}\right)
$$

where we have used $Y v=m_{e}$ as explained above. $M_{11}$ will be the upper leftmost entry in Eq. (18). Similarly, we get radiative correction to $M_{12,21}$, Fig. 5. Clearly, these are much smaller than $\bar{\lambda} w$. Other than providing a Majorana mass for the active neutrino $\nu_{e}$, they also transform the Dirac neutrino $N$ into a pseudo-Dirac one. Numerically, the splitting will be undetectably small for all practical purposes, and we can treat the $N$ as a Dirac neutrino. Assuming that there is no outstanding hierarchy between $m_{1}$ and $m_{S}$, then one expects the combination $w /\left(m_{1}^{2}-m_{S}^{2}\right) \ln \left(m_{1}^{1} / m_{S}^{2}\right) \simeq$ $w \sim \mathcal{O}(\mathrm{TeV})$. Plugging in the values, the resulting active neutrino mass is around $m_{\nu} \sim f Y_{1} \lambda_{1 \ell} \times 10^{3} \mathrm{eV}$. And the sub$\mathrm{eV}$ neutrino mass can be easily achieved with $f, Y_{1}, \lambda_{1 \ell} \sim$ $\mathcal{O}(0.1)$ without prominent fine-tuning.

\section{PHENOMENOLOGY OF $E, N$}

Even if the exotic leptons are too heavy to be produced by current or near-future colliders, they can have important effects at current energies. The notable ones are the electroweak oblique parameters $S, T[27,28]$ and the decay $h \rightarrow \gamma \gamma$.

\footnotetext{
${ }^{7}$ Our anomaly solutions can accommodate a variant of the type-I seesaw mechanism by adding a set of vectorlike SM singlet neutrinos $\mathcal{N}_{R}$ and $\mathcal{N}_{L}$ with lepton number unity. We shall not pursue this further.
} 


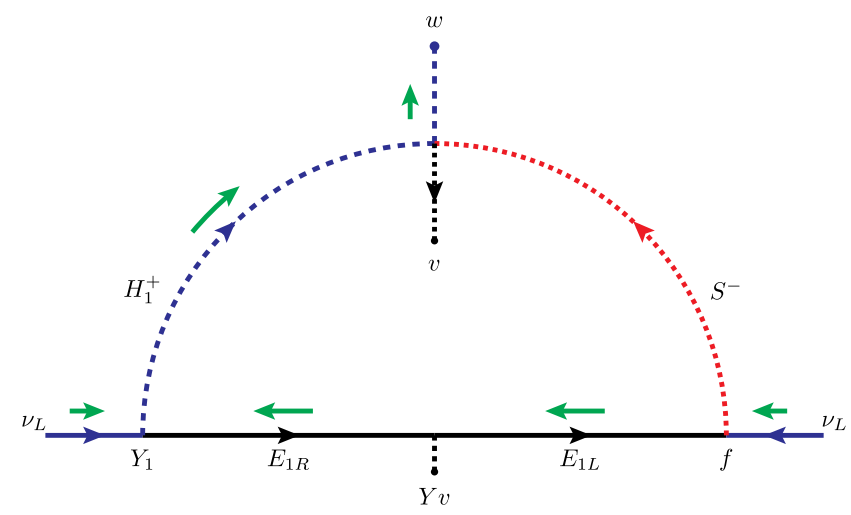

FIG. 4. One-loop $\nu_{e} \nu_{e}$ mass term generation. Green arrows show the flow of lepton charge.

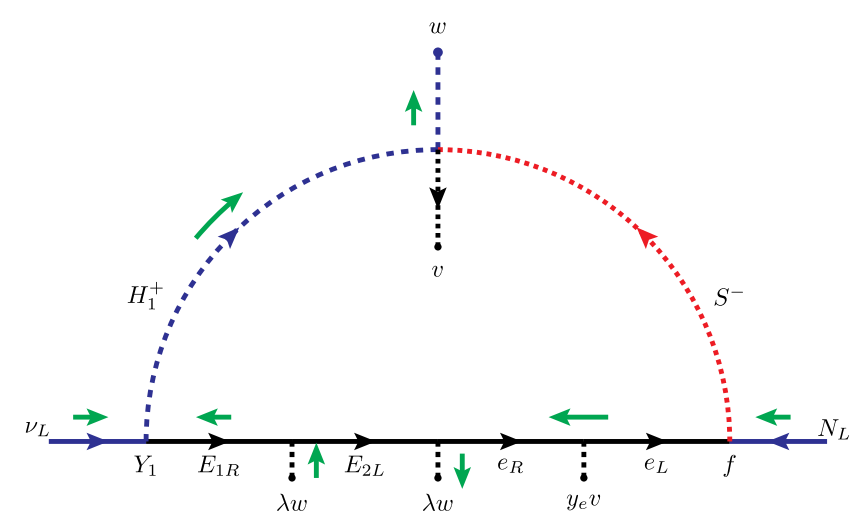

FIG. 5. One-loop $\nu_{e L} N$ mass term generations. Green arrows show the flow of lepton charge.

\section{A. Oblique parameters $S, T$}

It is well known that the oblique parameters $S$ and $T$ constraint heavy fermions that carry SM quantum numbers. In this case, they constrain the mass differences of the lepton pairs $E, N$ as well as the number of such pairs. Explicitly, for each generation, we have

$$
\begin{gathered}
\Delta T=\frac{1}{16 \pi s_{w}^{2}} \sum_{i=1,2} \frac{M_{E_{i}}^{2}}{M_{W}^{2}}\left(1+x_{i}+\frac{2 x_{i}}{1-x_{i}} \ln x_{i}\right), \\
\Delta S=\frac{1}{6 \pi} \sum_{i=1,2}\left(1+\ln x_{i}\right),
\end{gathered}
$$

where $x_{i}=M_{N_{i}}^{2} / M_{E_{i}}^{2}$. When the mass splitting between $E_{i}$ and $N_{i}$ is small compared to their masses,

$$
\begin{aligned}
\Delta T & \sim \frac{1}{12 \pi s_{w}^{2} M_{W}^{2}}\left[\left(M_{N_{1}}-M_{E_{1}}\right)^{2}+\left(M_{N_{2}}-M_{E_{2}}\right)^{2}\right], \\
\Delta S & \sim \frac{1}{3 \pi}\left[1+\frac{M_{N_{1}}-M_{E_{1}}}{M_{E_{1}}}+\frac{M_{N_{2}}-M_{E_{2}}}{M_{E_{2}}}\right],
\end{aligned}
$$

for each generation. The doublet $H_{1}$ provides the contribution

$$
\begin{gathered}
\Delta T=\frac{1}{16 \pi s_{w}^{2}} \frac{M_{H^{+}}^{2}}{M_{W}^{2}} \frac{1}{z}\left[1+z+\frac{2 z}{1-z} \ln z\right], \\
\Delta S=-\frac{1}{12 \pi} \ln z,
\end{gathered}
$$

where $z \equiv M_{H^{+}}^{2} / M_{H_{1}^{0}}^{2}$. Note that $\Delta T$ from the fermions and doublet scalar are both positive, but $\Delta S$ from the doublet scalar can be either positive or negative. From the Particle Data Group, we have $S_{\text {data }}<0.22$ and $T_{\text {data }}<0.27$ at 95\% C.L. [22].

To see how these will restrict the parameters of our model, we begin by taking $z=1$; i.e., the neutral and charged components of $H_{1}$ are degenerate. This implies that the mixing of $H_{1}$ with all other scalars is negligible. Then, the scalar contributions to $\Delta T$ and $\Delta S$ are vanishing. For simplicity, we also assume the masses of $E_{+}$and $E_{-}$are equal and their counterparts for $\mu$ and $\tau$ families are also the same. From Eq. (59), we see that the new isodoublet chiral leptons cannot have degenerate upper and lower components; otherwise, the resulting $\Delta S$ runs afoul of $S_{\text {data }}$. The splitting between the neutral and charged components that saturates $T_{\text {data }}$ is given by

$$
x=0.73 \text {, }
$$

where we have dropped the subscript $i$. Using Eq. (58) and $T_{\text {data }}$, we obtain $M_{E} \leq 350 \mathrm{GeV}$. The above values are to be taken as a demonstration that the stringent constraints of oblique corrections can be satisfied with new lepton masses in the range of $350 \mathrm{GeV}$, the vertical dash blue line in Fig. 6 . This is well above the limit from the charged lepton

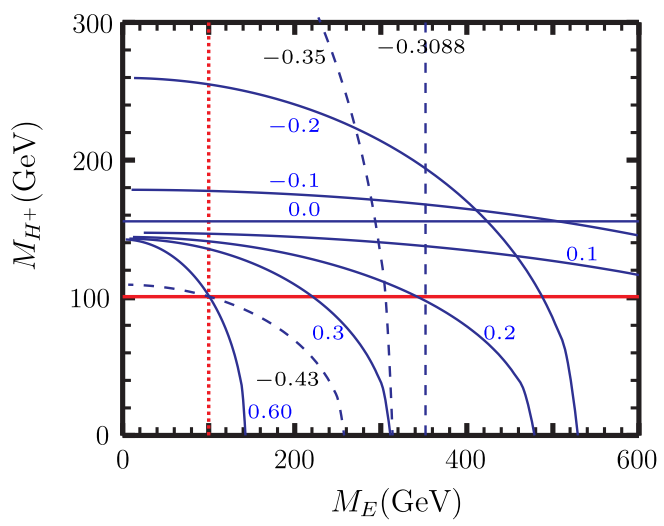

FIG. 6. Contours from $\Delta S$ and $\Delta T$ constraints for different $\ln x$. For a given $\ln x$, the allowed masses region is above the direct search bound on $M_{H^{+}}$, the horizontal red line, to the right of the direct search bound on $M_{E}$, the vertical dashed red line, and to the lower left of the blue (dashed) curve. 
searches of $>100.8 \mathrm{GeV}$, the vertical dash red line in Fig. 6, given by the Particle Data Group [22]. ${ }^{8}$

We can also take the limit at which the $E$ and $N$ are degenerate, i.e., all $x=1$. Then, Eq. (59) yields $\Delta S \simeq .32$. Then, it will require the $H_{1}$ doublet with $\ln z \simeq 3.71$ to bring it within the experimental bound since scalars give a negative contribution [see Eq. (62)]. The scalars will then be the sole contributors to $\Delta T$. A similar calculation gives an upper bound on the mass of $\mathrm{H}^{+}$, the horizontal blue line in Fig. 6. This value is also larger than the direct search bound on charged scalars, the horizontal red line in Fig. 6. The large splitting between $\mathrm{H}^{+}$and $H_{1}^{0}$ implies that some if not all of the parameters $\kappa_{H_{1} S}, \kappa_{\Phi_{i} H_{1}}$, and $\mu_{3}$ in Eq. (9) are large.

Notice that, since $z$ is large, we have $M_{H_{1}^{0}} \simeq 24.4 \mathrm{GeV}$. In general, it can mix with the SM Higgs boson, but we have seen that this mixing is constrained to be small. In the interaction basis, which is good for small mixings, it does not couple to quarks, and it has no couplings to gluons to one loop. Because of this, it will not run into problems at the LHC. Neutral scalars in the mass range of 10-100 GeV are notoriously difficult to detect. The challenges and a possible signal for probing this at the LHC were discussed in Ref. [30]. Additionally, a more promising avenue of exploring this at a future $e^{+} e^{-}$collider is given in Ref. [31].

From the above limiting case, it is easy to see that if $\ln x>$ -0.3088 the doublet $H_{1}$ will have to play a role in satisfying the oblique corrections bounds. For $\ln x=-0.3088$, it is the vertical dashed blue line in Fig. 6. It is more realistic to assume finite mass splittings between the isodoublets for both leptons and scalars. For an illustration, we take $\ln x=-0.2$; then, the masses of $M_{E}$ and $M_{H^{+}}$will satisfy a contour given by

$$
.0062\left(\frac{M_{E}}{M_{W}}\right)^{2}+.0259\left(\frac{M_{H^{+}}}{M_{W}}\right)^{2} \leq 0.27 .
$$

The allowed regions of $M_{E}$ and $M_{H^{+}}$for different $\ln x$ are displayed in Fig. 6. If both experimental lower bounds on $M_{E}, M_{H^{+}}$are met, one can see that the range of $\ln x$ is $-0.43572<\ln x<0.6053$, and it implies that $0.80<$ $\left(M_{N} / M_{E}\right)<1.35$ and $0.47<\left(M_{H^{+}} / M_{H^{0}}\right)<241.0$ if a universal $x_{i}$ is assumed. Moreover, for $\ln x>-0.1$, a light neutral scalar with mass $M_{H^{0}}<50 \mathrm{GeV}$ is expected.

Before closing this subsection, we remark that it is well known that the constraint from $\Delta T$ can be largely loosened by introducing an $S U(2)$ triplet with a small VEV so that the tree-level electroweak $\rho$ parameter is less than unity. However, for the minimal setup, we do not go further into such a discussion. See [32] for utilizing a triplet Higgs for neutrino mass generation and relaxing the $\Delta T$ constraint in this model.

\footnotetext{
${ }^{8}$ Note that the limit $M_{E} \gtrsim 168 \mathrm{GeV}$ at $95 \%$ C.L., obtained by Ref. [29], does not apply here since there is no tree-level $Z-E-l$ coupling in our model.
}

\section{B. Impact on Higgs decays}

\section{Higgs to two photons}

The SM Higgs to the diphoton vertex is generated at the one-loop level with dominant contributions from $W^{ \pm}$and top-quark running in the loop. In our model, there are six new charged leptons; a $E_{1}, E_{2}$ pair for each of the three generations; and two new charged scalars, $H_{1}^{ \pm}$and $S^{ \pm}$. These charged fields mix among themselves, and one needs to know every parameter for the actual mass diagonalization. However, with assumptions on the mass ranges of these charged particles, a general discussion is sufficient to draw qualitative conclusions.

In the mass basis, we can parametrize the Yukawa couplings and cubic couplings to the SM Higgs as

$$
\mathcal{L} \supset-\sum_{i=1}^{6} y_{E_{i}} \bar{E}_{i} E_{i} h-\sum_{i=1,2} \lambda_{i} M_{W} h H_{i}^{+} H_{i}^{-} .
$$

These new electrically charged degrees of freedom enter the one-loop triangle diagram and modify the width of the SM Higgs diphoton decay. This is given by [33]

$$
\begin{aligned}
\Gamma(H \rightarrow \gamma \gamma)= & \frac{G_{F} \alpha^{2} M_{H}^{3}}{128 \sqrt{2} \pi^{3}} \mid F_{1}\left(\tau_{W}\right)+\frac{4}{3} F_{1 / 2}\left(\tau_{t}\right) \\
& +\sum_{j=1,2} \lambda_{i} \frac{M_{W}^{2}}{g_{2} M_{H_{i}}^{2}} F_{0}\left(\tau_{H_{i}}\right) \\
& +\left.\sum_{i=1}^{6} y_{E_{i}} \frac{2 M_{W}}{g_{2} M_{E_{i}}} F_{1 / 2}\left(\tau_{E_{i}}\right)\right|^{2}
\end{aligned}
$$

where $\tau_{i} \equiv\left(m_{H} / 2 m_{i}\right)^{2}$, and

$$
\begin{aligned}
F_{0}(\tau) & =-[\tau-f(\tau)] / \tau^{2}, \\
F_{1 / 2}(\tau) & =2[\tau+(\tau-1) f(\tau)] / \tau^{2}, \\
F_{1}(\tau) & =-\left[2 \tau^{2}+3 \tau+3(2 \tau-1) f(\tau)\right] / \tau^{2},
\end{aligned}
$$

with

$$
f(\tau)= \begin{cases}{\left[\sin ^{-1} \sqrt{\tau}\right]^{2},} & \text { if } \tau \leq 1 \\ -\frac{1}{4}\left[\log \frac{1+\sqrt{1-1 / \tau}}{1-\sqrt{1-1 / \tau}}-i \pi\right]^{2}, & \text { if } \tau>1 .\end{cases}
$$

For $m_{i} \gg M_{H} / 2=62.5 \mathrm{GeV}$, we have the following expansions around $\tau=0$ : 


$$
\begin{aligned}
F_{0}(\tau) & \sim \frac{1}{3}+\frac{8}{45} \tau+\mathcal{O}\left(\tau^{2}\right), \\
F_{1 / 2}(\tau) & \sim \frac{4}{3}+\frac{14}{45} \tau+\mathcal{O}\left(\tau^{2}\right), \\
F_{1}(\tau) & \sim-7-\frac{22}{15} \tau+\mathcal{O}\left(\tau^{2}\right) .
\end{aligned}
$$

Plugging in the numbers, the diphoton decay width reads

$$
\begin{aligned}
\Gamma(H \rightarrow \gamma \gamma)= & \frac{G_{F} \alpha^{2} M_{H}^{3}}{128 \sqrt{2} \pi^{3}} \times \mid-8.324+1.834 \\
& +8.3 \times 10^{-4}\left(1.3 \times 10^{-2}\right) \times \lambda_{2}+0.087(0.42) \times \lambda_{1} \\
& +\sum_{i=1}^{6} 0.32(3.64) \times\left. y_{E_{i}}\right|^{2}
\end{aligned}
$$

for $M_{E_{i}}=1000(100) \mathrm{GeV}, M_{H_{2}}=2.0(0.5) \mathrm{TeV}$, and $M_{H_{1}}=200(100) \mathrm{GeV}$. The first two numbers are the dominant SM contributions from $W^{ \pm}$and the top quark, respectively. Since we expect $\left|y_{E_{i}}\right| \sim m_{l} / v_{h} \ll 1$, even for the new leptons (see Sec. III), the charged leptons contribution can be ignored. If the second charged scalar is heavy, its contribution can be ignored, too, even taking $\lambda_{2} \sim \mathcal{O}(1)$. Therefore, only the light charged scalar with mass in the range of 100 to $200 \mathrm{GeV}$ matters. The gluon fusion is the dominant SM Higgs production channel at the LHC, and it does not receive any modification. The signal strength of $p p \rightarrow h \rightarrow \gamma \gamma$ at the LHC is therefore

$$
\begin{aligned}
\mu_{\gamma \gamma} & \simeq \Gamma(H \rightarrow \gamma \gamma) / \Gamma(H \rightarrow \gamma \gamma)_{\mathrm{SM}} \\
& \sim 1-(0.03-0.13) \times \lambda_{1} .
\end{aligned}
$$

Even that the light charged Higgs has a coupling $\left|\lambda_{1}\right| \sim \mathcal{O}(1)$, the resulting di-photon signal strength still agrees with the experimental data $\mu_{\gamma \gamma}=1.18(+0.17-0.14)$ [34]. This is in agreement with the general analysis given in Ref. [35].

\section{Higgs to four fermions}

For notational simplicity, here, we denote $h_{1} \equiv \Re\left(H_{1}^{0}\right)$ and $a_{1} \equiv \Im\left(H_{1}^{0}\right)$. If $h_{1}$ (or $a_{1}$ ) is lighter than half the mass of the SM Higgs, $h_{\mathrm{SM}}$, then we can have

$$
h_{\mathrm{SM}} \rightarrow 2 h_{1}\left(2 a_{1}\right) \rightarrow \bar{\ell}_{i} \ell_{i}+\bar{\ell}_{j} \ell_{j} .
$$

The decay width is

$\Gamma\left(h_{\mathrm{SM}} \rightarrow h_{1} h_{1}\left(a_{1} a_{1}\right)\right)=\frac{v^{2}\left(\kappa_{2}+\kappa_{3}\right)^{2}}{32 \pi M_{H}}\left(1-\frac{4 m_{1}^{2}}{M_{H}^{2}}\right)^{\frac{1}{2}}$,

where $M_{H}(=125 \mathrm{GeV})$ and $m_{1}$ are the masses of $h_{\mathrm{SM}}$ and $h_{1}\left(a_{1}\right)$, respectively. We have neglected the term involving off-diagonal mixing of neutral scalars. The dominant decay mode for $h_{1}\left(a_{1}\right)$ is model dependent. The current bound on the mixing squared between $h_{\mathrm{SM}}$ and $h_{1}$ is about $\lesssim 10^{-2}$ for $10<m_{1}<40 \mathrm{GeV}$ from LEP2 [36]. ${ }^{9}$ For $Y_{1} \sim O(0.1)$, as expected from the radiative neutrino masses, the effects from the mixing with SM Higgs cannot compete with those from the direct Yukawa interaction, Eq. (19). Therefore, for scenario A, the main decay channel will be $h_{1}\left(a_{1}\right) \rightarrow \bar{\ell} \ell$ with $\ell=e, \mu, \tau$. The signal will be SM Higgs decays into two charged lepton pairs with both invariant masses peaking at the unknown $m_{1}$.

For scenario $\mathrm{B}, h_{1}\left(a_{1}\right)$ has only off-diagonal couplings to the SM lepton and a heavy lepton; see Eq. (23). The dominant decay of light $h_{1}\left(a_{1}\right)$ is expected to be due to mixing with the $h_{\mathrm{SM}}\left(\Im\left(H_{0}^{0}\right)\right)$, which then decays into a fermion pair. Therefore, $\bar{b} b$ will be the dominate final state if $m_{1}>10 \mathrm{GeV}$.

For the general case, in between scenarios $\mathrm{A}$ and $\mathrm{B}$, we expect the mixing element $\left|U_{12}\right|^{2}<0.13$, from unitarity and $\left|U_{11}\right|^{2}>0.87$ [15], to be small but nonvanishing.

\section{Colliders production and decay of exotic leptons}

For both scenarios $\mathrm{A}$ and $\mathrm{B}$, the $\mathrm{SM}$ gauge interaction allows $E_{ \pm} \rightarrow W^{-} N_{ \pm}, W^{-} N_{\mp}$, and $N_{+} \rightarrow N_{-} Z$ if $M_{N}<M_{E}$ is assumed. We consider the decay, $E_{ \pm} \rightarrow N W^{-}$, of a heavy Dirac $N$ for simplicity. The decay width is calculated to be

$$
\begin{aligned}
\Gamma_{E_{ \pm} \rightarrow N W^{-}} & =\frac{G_{F} M_{E}^{3}}{8 \sqrt{2} \pi} \lambda_{c m}\left(x_{N}, x_{w}\right) f\left(x_{w}, x_{N}\right) \\
f(x, y) & =x(1+y-2 x \mp 6 \sqrt{x})+(1-y)^{2},
\end{aligned}
$$

where $\lambda_{c m}(y, z)=\sqrt{1+y^{2}+z^{2}-2(y+z+y z)}, \quad x_{w}=$ $\left(M_{W} / M_{E}\right)^{2}$, and $x_{N} \equiv\left(M_{N} / M_{E}\right)^{2}$. For $M_{E} \gg M_{W}$, or $x_{w} \ll 1$, the width becomes

$$
\Gamma_{E_{ \pm} \rightarrow N W^{-}} \simeq \frac{G_{F} M_{E}^{3}}{8 \sqrt{2} \pi}\left(1-x_{N}\right)^{3} .
$$

As discussed in Sec. VII A, the oblique corrections require that $\ln x_{N}>-0.4357$, which implies that

$$
\Gamma_{E_{ \pm} \rightarrow N W^{-}}<14.45 \times\left(\frac{M_{E}}{1 \mathrm{TeV}}\right)^{3} \mathrm{GeV} .
$$

On the other hand, if $M_{N}>M_{E}$, the decay width of $N$ takes a similar form with $M_{E} \leftrightarrow M_{N}$,

$$
\Gamma_{N \rightarrow E_{ \pm} W^{+}} \simeq \frac{G_{F} M_{N}^{3}}{8 \sqrt{2} \pi}\left(1-x_{N}^{-1}\right)^{3} .
$$

Similarly, from $\ln x_{N}<0.6053$,

\footnotetext{
${ }^{9}$ The limit on the mixing squared between $h_{\mathrm{SM}}$ and $h_{1}$ could be improved by a few orders of magnitude at the future colliders $[30,31]$.
} 
TABLE IV. The $H_{1}$ Yukawa couplings between the heavy leptons and the SM ones in our model. The heavy neutrino is assumed to be Dirac.

\begin{tabular}{lccccc}
\hline \hline Scenario & $F$ & $f$ & $\phi$ & $s$ & $a$ \\
\hline \multirow{4}{*}{$\mathrm{A}$} & $E_{ \pm}$ & $e$ & $h_{1}$ & 0 & $-\frac{Y_{1}}{2 \sqrt{2}}$ \\
& $E_{ \pm}$ & $e$ & $a_{1}$ & $-\frac{i Y_{1}}{2 \sqrt{2}}$ & 0 \\
& $E_{ \pm}$ & $\nu$ & $H_{1}^{-}$ & $\frac{Y_{1}}{4 \sqrt{2}}$ & $-\frac{Y_{1}}{4 \sqrt{2}}$ \\
& $N$ & $e$ & $H_{1}^{+}$ & $-\frac{Y_{1}}{4}$ & $-\frac{Y_{1}}{4}$ \\
& $E_{ \pm}$ & $e$ & $h_{1}$ & $\frac{Y_{1}}{4}$ & $-\frac{Y_{1}}{4}$ \\
$\mathrm{~B}$ & $E_{ \pm}$ & $e$ & $a_{1}$ & $-\frac{i Y_{1}}{4}$ & $\frac{i Y_{1}}{4}$ \\
& $E_{ \pm}$ & $\nu$ & $H_{1}^{-}$ & $\frac{Y_{1}}{2 \sqrt{2}}$ & $-\frac{Y_{1}}{2 \sqrt{2}}$ \\
\hline \hline
\end{tabular}

$$
\Gamma_{N \rightarrow E_{ \pm} W^{-}}<30.72 \times\left(\frac{M_{N}}{1 \mathrm{TeV}}\right)^{3} \mathrm{GeV}
$$

From the above discussion, unless the leptons are nearly degenerate, the decays of $E_{ \pm}$or $N$ are expected to be prompt.

Next, we turn our attention to the heavy lepton decay via the Yukawa interaction with $H_{1}$. Let us consider a general case with two fermions $F$ and $f$ and a scalar $\phi$. The scalar field $\phi$ could be either neutral or charged. Assume that they admit a Yukawa interaction that is parametrized as $\mathcal{L} \supset$ $\bar{F}\left(s+a \gamma^{5}\right) f \phi+$ H.c.. If kinematics is allowed, the decay channel $F \rightarrow f \phi$ opens, and the width is calculated to be

$\frac{\lambda_{c m}\left(x_{f}, x_{\phi}\right) M}{16 \pi}\left[\left(1+x_{f}\right)^{2}|s|^{2}+\left(1-x_{f}\right)^{2}|a|^{2}-x_{\phi}\left(|s|^{2}+|a|^{2}\right)\right]$,

where $M$ is the mass of $F, x_{f}=\left(m_{f} / M\right)^{2}$, and $x_{\phi}=\left(m_{\phi} / M\right)^{2}$. For the cases in which $M, m_{f} \gg m_{\phi}$, the decay width becomes

$\Gamma(F \rightarrow f \phi) \simeq \frac{M}{16 \pi}\left(1-x_{f}\right)\left[\left(1+x_{f}\right)^{2}|s|^{2}+\left(1-x_{f}\right)^{2}|a|^{2}\right]$.

The relevant fields and Yukawa couplings in our model are collected and listed in Table IV. One can read the precise expression by using Eq. (80) and Table IV. Roughly speaking, the decay widths are about $\sim\left(Y_{1}^{2} / 64 \pi\right) M$, or numerically $\sim 0.05 \times\left(Y_{1} / 0.1\right)^{2} \times(M / 1 \mathrm{TeV}) \mathrm{GeV}$, where $M$ is the mass of $E_{ \pm}$or $N$. In general, this decay width is much smaller than that from the decay with a SM $W^{ \pm}$boson in the final states. Note that in scenario B $N$ does not have the tree-level two-body decays via the Yukawa interaction with $H_{1} \cdot{ }^{10}$ However, if the mass of the charged scalar $S^{ \pm}$is less

\footnotetext{
${ }^{10}$ We have checked that even $M_{N}<M_{E} N$ cannot be a dark matter candidate due to its SM $S U(2)$ interaction. Adding an ad hoc $Z_{2}$ parity will not change this.
}

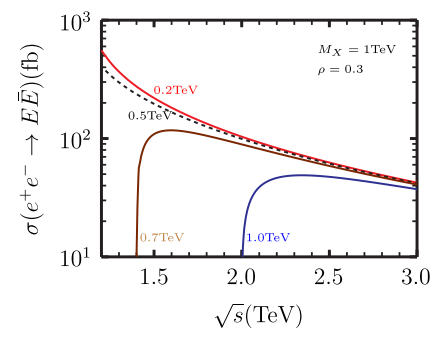

(a)

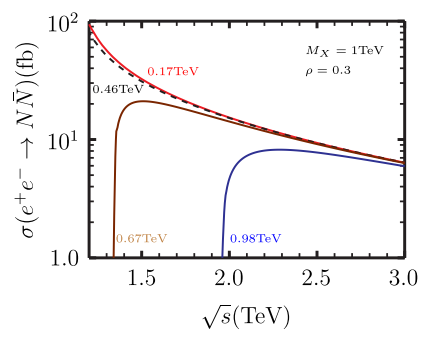

(b)
FIG. 7. (a) The $E \bar{E}$ and (b) $N \bar{N}$ production cross sections vs $\sqrt{s}$ at an $e^{+} e^{-}$collider. The masses of $E(N)$ are labeled next to the curves.

than $M_{N}$, then the decay $N \rightarrow e^{+} S^{-11}$ is possible. Otherwise, $N$ will have only three-body decays. For $M_{N}<M_{E}$, there is another chain with an intermediate virtual $E$; for example, $N \rightarrow W^{+} E^{*} \rightarrow W^{+} h_{1} e^{-}$. We shall use the superscript $*$ to denote off-shell particles.

The heavy leptons can be pair produced at the $e^{+} e^{-}$ colliders. For simplicity, we assume that $E_{+}$and $E_{-}$are nearly degenerate so that they are hard to distinguish experimentally, and we collectively denote the states as $E \equiv E_{+} \sim E_{-}$. For $\sqrt{s} \gg M_{Z}$ and away from the $Z_{\ell}$ pole, the production cross section per generation for scenario $\mathrm{B}$ can be calculated to be

$$
\begin{aligned}
& \sigma\left(e^{+} e^{-} \rightarrow E \bar{E}\right) \\
& \simeq 2 \frac{4 \pi \alpha^{2}}{3 s} \sqrt{1-4 x_{E}}\left\{\left(1+\frac{\rho s}{s-M_{X}^{2}}\right)^{2}\left(1+2 x_{E}\right)\right. \\
& \quad+\frac{\left(g_{L}^{e}\right)^{2}+\left(g_{R}^{e}\right)^{2}}{4\left(s_{W} c_{W}\right)^{4}}\left[\left(1-x_{E}\right)\left(\left(g_{L}^{E}\right)^{2}+\left(g_{R}^{E}\right)^{2}\right)+6 x_{E} g_{L}^{E} g_{R}^{E}\right] \\
& \left.\quad+\frac{\left(g_{L}^{e}+g_{R}^{e}\right)}{2\left(s_{W} c_{W}\right)^{2}}\left(g_{L}^{E}+g_{R}^{E}\right)\left(1+\frac{\rho s}{s-M_{X}^{2}}\right)\left(1-x_{E}\right)\right\},
\end{aligned}
$$

where $x_{E} \equiv M_{E}^{2} / s, g_{L}^{E} \simeq g_{L}^{e}=-1 / 2+s_{W}^{2}$, and $g_{R}^{E} \simeq g_{R}^{e}=$ $s_{W}^{2}$. The first factor 2 represents the incoherent sum from the contribution of the two heavy Dirac charged leptons. For example, if $\left\{M_{E_{+}}, M_{E_{-}}\right\}=\{200,180\} \mathrm{GeV}, \rho=0.3$, and $M_{X}=1 \mathrm{TeV}$, the production cross section of two charged leptons is 556.0 (83.2) fb for $\sqrt{s}=1.2$ (2.2) TeV; see Fig. 7(a). Similarly, the heavy $N$ can be pair produced through the s-channel process mediated by $Z, Z_{\ell}$, and the production cross section for each generation is

\footnotetext{
${ }^{11}$ Because of the radiative generated Majorana masses, Fig. 5, $N$ is in fact pseudo-Dirac. However, the conjugate decays, $N \rightarrow$ $W^{-} \bar{E}$ and $N \rightarrow e^{-} S^{+}$, are expected to be rare.
} 
TABLE V. The production cross sections(in pb) at the LHC14 for one generation in scenario-B in our model. The masses of $E_{ \pm}$ are in the unit of $\mathrm{GeV}$.

\begin{tabular}{cccccc}
\hline \hline & $M_{E_{+}}$ & $M_{E_{-}}$ & $\bar{E}_{+} E_{+}$ & $\bar{E}_{+} E_{-}+\bar{E}_{-} E_{+}$ & $\bar{E}_{-} E_{-}$ \\
\hline set-1 & 200 & 180 & $9.62 \times 10^{-2}$ & $4.27 \times 10^{-2}$ & $1.40 \times 10^{-1}$ \\
set-2 & 500 & 480 & $2.76 \times 10^{-3}$ & $9.04 \times 10^{-4}$ & $3.30 \times 10^{-3}$ \\
set-3 & 1000 & 950 & $9.08 \times 10^{-5}$ & $2.44 \times 10^{-5}$ & $1.21 \times 10^{-4}$ \\
\hline \hline
\end{tabular}

$$
\begin{aligned}
\sigma\left(e^{+} e^{-} \rightarrow N \bar{N}\right) \\
\simeq \frac{4 \pi \alpha^{2}}{3 s} \sqrt{1-4 x_{N}}\left\{\left(\frac{\rho s}{s-M_{X}^{2}}\right)^{2}\left(1+2 x_{N}\right)\right. \\
\quad+\frac{\left(g_{L}^{e}\right)^{2}+\left(g_{R}^{e}\right)^{2}}{8\left(s_{W} c_{W}\right)^{4}}\left(1+2 x_{N}\right) \\
\left.\quad+\frac{\left(g_{L}^{e}+g_{R}^{e}\right)}{2\left(s_{W} c_{W}\right)^{2}}\left(\frac{\rho s}{s-M_{X}^{2}}\right)\left(1-x_{N}\right)\right\} .
\end{aligned}
$$

For example, if $M_{N}=170 \mathrm{GeV}, \quad \rho=0.3, \quad$ and $M_{X}=1 \mathrm{TeV}$, the production cross section of the $N \bar{N}$ pair is 94.0 (12.4) fb for $\sqrt{s}=1.2(2.2) \mathrm{TeV}$; see Fig. 7(b).

At the LHC, the heavy leptons can be produced via the photon and/or the $W$-/Z-boson Drell-Yan process. Therefore, the production cross sections are independent of the $Z_{\ell}$ mass and the $U(1)_{\ell}$ gauge couplings. Since a fullfledged collider study is beyond the scope of this paper, only the production cross sections are considered here. Three sets of $\left(M_{E_{+}}, M_{E_{-}}\right)$are considered as the benchmarks with the assumption that their mass differences are subelectroweak. For each benchmark, $M_{N}$ is chosen to meet the constraint from the electroweak precision; with $|\ln x|<$ $0.3,0.15,0.05$ for $M_{E_{+}}=200,500,1000 \mathrm{GeV}$, respectively. The production cross sections are evaluated with CALCHEP and are listed in Tables V and VI.

For $M_{E}, M_{N} \lesssim 500 \mathrm{GeV}$, the production cross sections are about $\mathcal{O}(1-100) \mathrm{fb}$. The production of $E$ and $N$ will be followed by their decays into SM particles. The decay modes are sensitive to the masses of $E_{+}, E_{-}, N$ as well as the masses of the charged scalars $H_{1}^{ \pm}$and $S^{ \pm}$, which in general mix. If the splitting between $E$ and $N$ is large

TABLE VI. The production cross sections(in pb) at the LHC14 for one generation in scenario-B in our model. The masses of $N$ are in the unit of $\mathrm{GeV}$.

\begin{tabular}{lcccc}
\hline \hline & $M_{N}$ & $\bar{N} N$ & $\bar{N} E_{+}+\bar{E}_{+} N$ & $\bar{N} E_{-}+\bar{E}_{-} N$ \\
\hline set-1 & 170 & $3.68 \times 10^{-1}$ & $1.19 \times 10^{-1}$ & $5.20 \times 10^{-2}$ \\
set-1 & 230 & $1.21 \times 10^{-1}$ & $6.90 \times 10^{-2}$ & $2.89 \times 10^{-2}$ \\
set-2 & 460 & $7.76 \times 10^{-3}$ & $3.04 \times 10^{-3}$ & $1.04 \times 10^{-3}$ \\
set-2 & 540 & $3.87 \times 10^{-3}$ & $2.17 \times 10^{-3}$ & $7.24 \times 10^{-4}$ \\
set-3 & 980 & $1.93 \times 10^{-4}$ & $9.28 \times 10^{-5}$ & $2.69 \times 10^{-5}$ \\
set-3 & 1020 & $1.53 \times 10^{-4}$ & $8.29 \times 10^{-5}$ & $2.37 \times 10^{-5}$ \\
\hline \hline
\end{tabular}

enough, the dominant decays will be two-body modes; otherwise, they will be three-body modes. They also depend on the ordering of the $E$ and $N$ masses. If $M_{E}>M_{S^{ \pm}}$, then we have the chain

$$
\begin{aligned}
E \rightarrow \bar{\nu}+ & S^{-} \\
& \left\llcorner W^{-}+h_{1}\left(a_{1}\right),\right.
\end{aligned}
$$

where the decay of $S^{-}$proceeds via mixing with $H_{1}^{-}$. Additionally, in scenario B, if $M_{E}>M_{N}$, we also have the chain

$$
\begin{gathered}
E \rightarrow W^{-}+N \\
\downarrow \\
W^{-}+E^{(*)}+W^{+} \\
\quad e^{-}+h_{1}\left(a_{1}\right),
\end{gathered}
$$

where we take the decay of $E$ to proceed via

$$
E \rightarrow e+h_{1}\left(a_{1}\right) \rightarrow e+\ell \bar{\ell}
$$

if $Y_{1}$ is not too small.

Next, we examine the decays of the neutral lepton $N$. If $M_{N}>M_{E}$, the decay chain will be

$$
\begin{aligned}
N \rightarrow W^{+}+ & E \\
& \sqcup e^{-}+h_{1}\left(a_{1}\right) .
\end{aligned}
$$

Similar to the case of $E$, if $M_{N}>M_{S^{ \pm}}$, the following is also available:

$$
\begin{aligned}
N \rightarrow e^{+}+ & S^{-} \\
& \left\llcorner W^{-} h_{1}\left(a_{1}\right) .\right.
\end{aligned}
$$

Before one can draw any conclusion, it is crucially important to understand the SM background first. We leave the comprehensive signal and background study to a future work.

\section{CONCLUSIONS}

An anomaly-free gauged $U(1)_{\mathfrak{l}}$ lepton model was constructed to study the nature of the lepton number. Differently from previous studies in the literature, we found two solutions that are free of the anomaly for each SM fermion generation. Our solutions also do not require the type-I seesaw mechanism for active neutrino mass generation. The price we pay is introducing four extra chiral fermion fields per generation, while the two solutions, of which anomaly cancelation is nontrivial, look superficially similar. The fermion content of one solution is displayed in Table I. We have constructed the minimal scalar sector, Table II, such that the active neutrino masses are generated radiatively without significantly fine tuning 
the model parameters. Moreover, the new leptons acquire their masses from the vacuum expectation values of SM Higgs doublet and the scalars, $\phi_{1,2}$, which carry nonzero lepton numbers.

An immediate phenomenological consequence is the existence of a new gauge boson, $Z_{\mathfrak{l}}$, which is universal for any gauged $U(1)_{\ell}$ model. The mass of $Z_{\mathfrak{l}}, M_{X}$, is determined by the lepton charges of the scalars $\phi_{1,2}$ and the lepton number-violating VEVs, $\left\langle\phi_{1,2}\right\rangle$. The $Z_{\mathfrak{l}}$ boson interferes with the SM photon and $Z$ boson in the process $e^{+} e^{-} \rightarrow l \bar{l}$. Even if the center-of-mass energy of the $e^{+} e^{-}$ collider is below the mass of $Z_{\ell}$, its effects can be unambiguously identified from the $Z$-line shape and the forward-backward asymmetries. This can be seen in Figs. 1 and 2. In contrast, the front-back asymmetries of the quarks will not change from their SM values. The combination of these two measurements will shed light on the nature of any extra $Z$ boson.

As noted previously, $Z_{\mathfrak{l}}$ can be produced at a hadron collider by radiating from a lepton line from the usual Drell-Yan process via the reaction $p p \rightarrow \ell \bar{\ell} \ell^{\prime} \bar{\ell}^{\prime}$ with the invariant mass of one pair of leptons, $\ell \bar{\ell}^{\prime}$, say, peaking at $M_{X}$. The final state to look for is four leptons with no jets. We found that the LHC14, LHC30, and LHC100 with an integrated luminosity $3000(f \mathrm{~b})^{-1}$ can probe the lepton number-violating scale up to roughly $0.5,1.0$, and $2.0 \mathrm{TeV}$, respectively. This is the same range of direct $Z_{\mathfrak{l}}$ production can be reached at the $e^{+} e^{-}$colliders such as ILC500 and CLIC at $2 \mathrm{TeV}$. The latter also provides a much cleaner environment. For the constraint on the coupling for a light scenario B-type $Z_{\ell}$, see Ref. [37].

Since the SM fermions content is anomalous under $U(1)_{\ell}$, new heavy leptons are mandated to cancel the anomaly for a UV-complete theory. The masses of these exotic leptons are usually free parameters and can be heavier than the reach of any foreseeable colliders. We studied the phenomenologically more interesting case in which their masses are $<1.0 \mathrm{TeV}$. The production of the heavy charged lepton pair at an $e^{+} e^{-}$collider is $\sim O\left(10-10^{2}\right) \mathrm{fb}$, and the signals for their detection can be very clean. Because of the negligible mixing of $E$ and $N$ with their SM counterparts, the usual detection channels do not apply. For example, for a heavy neutrino $N$, the usual detection channel is $N \rightarrow e W$. However, for our solution, $N$ predominantly decays into final states with $W+3 \ell$ [see Eqs. (86) and (87)]. We have used the result that $h_{1}\left(a_{1}\right)$ will have sizable couplings to SM charged leptons with $Y_{1} \gtrsim 0.1$.

The production and detection at a hadron collider are much more complicated. While the production cross sections are not too small at the LHC, i.e., $\mathcal{O}\left(1-10^{2}\right)$ fb for $M_{E}, M_{N}<$ $500 \mathrm{GeV}$, one needs a comprehensive study of the SM backgrounds for each possible final state. But one needs a comprehensive study of the SM backgrounds for each possible final state. For a heavy neutrino with the usual decay, this has been extensively studied before [38]. Our preferred final states are different and typically involve multileptons and no accompany jet activities other than hadronic $W$. We shall leave such a comprehensive study to future work. We note that multilepton signals at the LHC were investigated in Refs. [39-41] for various scenarios and different models.

Moreover, we have also studied the imprints of the new scalars and heavy leptons at low energies. It is found that the most prominent constraint on the new fields is from the oblique parameters, $\Delta S$ and $\Delta T$. We have carefully studied the current experimental bounds on $\Delta S$ and $\Delta T$ and the direct searches for the new charged scalar and heavy charged lepton as well. The electroweak precision bounds require that the new heavy leptons have to be nearly degenerate. In any gauged $U(1)_{\mathfrak{r}}$ model with the custodial symmetry, the approximate mass degeneracy of the new $S U(2)$ doublet leptons is a generic feature. Depending on their masses, the mass splitting between the heavy neutrino and charged lepton has to be less than $\mathcal{O}(1 \%-10 \%)$. This will severely constrain the parameters of the model. We look forward to future improvements on the measurements of these quantities.

\section{ACKNOWLEDGMENTS}

W. F. C. is supported by the Taiwan Ministry of Science and Technology under Grants No. 106-2112-M-007-009MY3 and No. 105-2112-M-007-029. W. F. C. is thankful for the hospitality of Professor Chi-Ting Shih and the Department of Applied Physics of Tunghai University where part of his work was done. TRIUMF receives federal funding via a contribution agreement with the National Research Council of Canada and the Natural Science and Engineering Research Council of Canada.
[1] S. Weinberg, Phys. Rev. Lett. 43, 1566 (1979).

[2] S. L. Glashow, NATO Sci. Ser. B 61, 687 (1980); T. Yanagida, Conf. Proc., C7902131 (1979) 95; R. N. Mohapatra and G. Senjanovic, Phys. Rev. Lett. 44, 912 (1980); J. Schechter and J. W. F. Valle, Phys. Rev. D 25, 774 (1982).
[3] W.-F. Chang, J. N. Ng, and J. M. S. Wu, Phys. Lett. B 730, 347 (2014).

[4] W.-F. Chang and J. N. Ng, Phys. Rev. D 90, 065034 (2014).

[5] W.-F. Chang and J. N. Ng, J. Cosmol. Astropart. Phys. 07 (2016) 027. 
[6] T. D. Lee and C.-N. Yang, Phys. Rev. 98, 1501 (1955).

[7] L. Okun, Phys. Lett. B 382, 389 (1996).

[8] P. Fileviez Perez and M. B. Wise, Phys. Rev. D 82, 011901 (2010); 82, 079901(E) (2010).

[9] P. Schwaller, T. M. P. Tait, and R. Vega-Morales, Phys. Rev. D 88, 035001 (2013).

[10] M. Magg and C. Wetterich, Phys. Lett. 94B, 61 (1980); G. Lazarides, Q. Shafi, and C. Wetterich, Nucl. Phys. B181, 287 (1981); R. N. Mohapatra and G. Senjanovic, Phys. Rev. D 23, 165 (1981); T. P. Cheng and L.-F. Li, Phys. Rev. D 22, 2860 (1980).

[11] W. Chao, Phys. Lett. B 695, 157 (2011).

[12] B. Fornal, Y. Shirman, T. M. P. Tait, and J. R. West, Phys. Rev. D 96, 035001 (2017).

[13] B. Holdom, Phys. Lett. 166B, 196 (1986); Phys. Lett. B 259, 329 (1991).

[14] W.-F. Chang, J. N. Ng, and J. M. S. Wu, Phys. Rev. D 74, 095005 (2006); 79, 039902(E) (2009).

[15] G. Aad et al. (ATLAS and CMS Collaborations), J. High Energy Phys. 08 (2016) 045.

[16] H. Baer et al., arXiv:1306.6352.

[17] P. Lebrun, L. Linssen, A. Lucaci-Timoce, D. Schulte, F. Simon, S. Stapnes, N. Toge, H. Weerts, and J. Wells, arXiv:1209.2543.

[18] S. Schael et al. (DELPHI, OPAL, LEP Electroweak, ALEPH, and L3 Collaborations), Phys. Rep. 532, 119 (2013).

[19] A. Leike, T. Riemann, and J. Rose, Phys. Lett. B 273, 513 (1991); T. Riemann, Phys. Lett. B 293, 451 (1992).

[20] M. Bicer et al. (TLEP Design Study Working Group Collaboration), J. High Energy Phys. 01 (2014) 164; D. d'Enterria, Frascati Phys. Ser. 61, 17 (2016).

[21] CEPC-SPPC Study Group, CEPC-SPPC Preliminary Conceptual Design Report. 1. Physics and Detector, 2015.

[22] C. Patrignani et al. (Particle Data Group Collaboration), Chin. Phys. C 40, 100001 (2016).
[23] M. Lindner, M. Platscher, and F. S. Queiroz, Phys. Rep. 731, 1 (2018).

[24] P. L. Anthony et al. (SLAC E158 Collaboration), Phys. Rev. Lett. 95, 081601 (2005).

[25] A. Belyaev, N. D. Christensen, and A. Pukhov, Comput. Phys. Commun. 184, 1729 (2013).

[26] J. Pumplin, D. R. Stump, J. Huston, H. L. Lai, P. M. Nadolsky, and W. K. Tung, J. High Energy Phys. 07 (2002) 012.

[27] M. E. Peskin and T. Takeuchi, Phys. Rev. Lett. 65, 964 (1990).

[28] M. E. Peskin and T. Takeuchi, Phys. Rev. D 46, 381 (1992).

[29] G. Aad et al. (ATLAS Collaboration), J. High Energy Phys. 09 (2015) 108.

[30] W.-F. Chang, T. Modak, and J. N. Ng, Phys. Rev. D 97, 055020 (2018).

[31] W.-F. Chang, J. N. Ng, and G. White, Phys. Rev. D 97, 115015 (2018).

[32] W. F. Chang and J. N. Ng, arXiv:1807.09439.

[33] A. Djouadi, Phys. Rep. 457, 1 (2008).

[34] A. M. Sirunyan et al. (CMS Collaboration), arXiv:1804.02716.

[35] W.-F. Chang, J. N. Ng, and J. M. S. Wu, Phys. Rev. D 86, 033003 (2012).

[36] R. Barate et al. (OPAL, DELPHI, LEP Working Group for Higgs Boson Searches, ALEPH, and L3 Collaborations), Phys. Lett. B 565, 61 (2003).

[37] Y. S. Jeong, C. S. Kim, and H.-S. Lee, Int. J. Mod. Phys. A 31, 1650059 (2016).

[38] J. N. Ng, A. de la Puente, and B. W. -P. Pan, J. High Energy Phys. 12 (2015) 1.

[39] J. A. Aguilar-Saavedra, Nucl. Phys. B828, 289 (2010).

[40] C.-Y. Chen and P.S. B. Dev, Phys. Rev. D 85, 093018 (2012).

[41] N. Arkani-Hamed, K. Blum, R. T. D'Agnolo, and J. Fan, J. High Energy Phys. 01 (2013) 149. 